\title{
Triality in little string theories
}

\author{
Brice Bastian, ${ }^{1, *}$ Stefan Hohenegger, ${ }^{1, \dagger}$ Amer Iqbal, ${ }^{2,3, *}$ and Soo-Jong Rey ${ }^{4, \S}$ \\ ${ }^{1}$ Université de Lyon UMR 5822, CNRS/IN2P3, Institut de Physique Nucléaire de Lyon 4 rue Enrico Fermi, \\ 69622 Villeurbanne Cedex, France \\ ${ }^{2}$ Abdus Salam School of Mathematical Sciences Government College University, Lahore 54660, Pakistan \\ ${ }^{3}$ Center for Theoretical Physics, Lahore 54660, Pakistan \\ ${ }^{4}$ School of Physics and Astronomy \& Center for Theoretical Physics Seoul National University, \\ Seoul 08826 Korea
}

(Received 6 December 2017; published 9 February 2018)

\begin{abstract}
We study a class of eight-supercharge little string theories (LSTs) on the world volume of $N$ M5-branes with transverse space $\mathbb{S}^{1} \times\left(\mathbb{C}^{2} / \mathbb{Z}_{M}\right)$. These M-brane configurations compactified on a circle are dual to $M$ D5-branes intersecting $N$ NS5-branes on $\mathbb{T}^{2} \times \mathbb{R}^{7,1}$ as well as to F-theory compactified on a toric CalabiYau threefold $X_{N, M}$. We argue that the Kähler cone of $X_{N, M}$ admits three regions associated with weakly coupled quiver gauge theories of gauge groups $[U(N)]^{M},[U(M)]^{N}$, and $\left[U\left(\frac{N M}{k}\right)\right]^{k}$ where $k=\operatorname{gcd}(N, M)$. These provide low-energy descriptions of different LSTs. The duality between the first two gauge theories is well known and is a consequence of the S-duality between D5- and NS5-branes or the T-duality of the LSTs. The triality involving the third gauge theory is new, and we demonstrate it using several examples. We also discuss implications of this triality for the W-algebras associated with the Alday-GaiottoTachikawa dual theories.
\end{abstract}

DOI: 10.1103/PhysRevD.97.046004

\section{INTRODUCTION}

Throughout the years, dualities have been a driving tool in the exploration and development of statistical physics, quantum field theory, and (non)perturbative string theory. Either as conceptual means to analyze the (mathematical) structure of the theory or as computational tools to reformulate specific questions in a more tractable framework, dualities were proven extremely useful and, in many cases, have been at the forefront of new discoveries. While new dualities (or associated symmetries) are typically difficult to find (or prove) in full-fledge string theories, they typically also leave imprints on other physical system that are engineered by string (or M-) theory. One such example is that of little string theories (LSTs) [1-7] (see [8,9] for a review): using various brane constructions (in string or M-theory) and their dual geometric description in F-theory [10], different realizations of LSTs can be constructed and duality relations among them can be studied

\footnotetext{
*b.bastian@ipnl.in2p3.fr

†s.hohenegger@ipnl.in2p3.fr

*amer@alum.mit.edu

sjrey@snu.ac.kr
}

Published by the American Physical Society under the terms of the Creative Commons Attribution 4.0 International license. Further distribution of this work must maintain attribution to the author(s) and the published article's title, journal citation, and DOI. Funded by SCOAP ${ }^{3}$.
[11,12], including heterotic LSTs [13-15]. In this paper, we aim to further analyze the web of dualities connecting different LSTs as well as their low energy descriptions in terms of gauge field theories.

LSTs refer to a class of interacting, ultraviolet-complete quantum theories in six dimensions whose nonlocal dynamics is governed by self-dual noncritical strings. While being easier to handle due to the fact that gravity is decoupled (i.e., the spectrum does not contain a massless spin-two field), these theories still share many features in common with the critical string theory in ten dimensions. In fact, LSTs are operationally definable from type II string theory (or its dual avatars) through a particular decoupling limit that sends the string coupling constant to zero $\left(g_{\mathrm{st}} \rightarrow 0\right)$ while at the same time keeping the string length $\ell_{\text {st }}$ finite. Depending on details of the original type II setup, one can construct various different LSTs with $(2,0)$ or $(1,0)$ supersymmetry.

The construction of LSTs which will be relevant for us is in terms of F-theory compactifications [16-21] on a class of toric, noncompact Calabi-Yau threefolds called $X_{N, M}$ : the latter are equipped with the structure of a double elliptic fibration, in which one elliptic fibration has a singularity of type $I_{N-1}$ and the other one of $I_{M-1}$ $[22,23]$. These LSTs of type $(N, M)$ can also be engineered using $N$ parallel M5-branes with a transverse orbifold of type $A_{M-1}$ [24-27]. Moreover, using the refined topological vertex formalism [28], the topological string partition 
function $\mathcal{Z}_{N, M}\left(\mathbf{T}, \mathbf{t}, m, \epsilon_{1}, \epsilon_{2}\right)$ of $X_{N, M}$ has been computed in $[12,27,29]$ at a particular region of the Käher moduli space: in this region $\mathbf{T}=\left\{T_{1}, \ldots, T_{M}\right\}$ and $\mathbf{t}=\left\{t_{1}, \ldots, t_{N}\right\}$ are two sets of Kähler parameters related to the two elliptic fibrations of $X_{N, M}$, respectively (and $m$ is a further Kähler modulus), while $\epsilon_{1,2}$ are related to the topological string coupling and the beta deformation due to the $\Omega$ background [30-32]. $\mathcal{Z}_{N, M}(\mathbf{T}, \mathbf{t}, m, \epsilon,-\epsilon)$ have also been studied for quantized values of the parameters $\mathbf{t}$ in units of $\epsilon$ and shown to be given by highest weight representations of an affine algebra [33]. It was proposed in [12] that the topological string partition function $\mathcal{Z}_{N, M}$ captures the partition functions of a class of LST with 8 supercharges. Compactification of LSTs of type $(N, M)$ on a circle then relates these with LSTs of type $(M, N)$ by T-duality. This T-duality relation is reflected in the Calabi-Yau $X_{N, M}$ by the exchange of the two fibers $[10,22]$. See $[34,35]$ for discussion of topological string partition functions on elliptically fibered Calabi-Yau threefolds.

Furthermore, the low energy limits of the compactified LSTs of type $(N, M)$ and $(M, N)$ are the (circular) quiver gauge theories with gauge group $[U(N)]^{M}$ and $[U(M)]^{N}$, respectively. In these two descriptions, the parameters $\mathbf{T}$ and $\mathbf{t}$ are interpreted as either the gauge coupling constants or the Coulomb-branch parameters (while $m$ is interpreted as the mass scale of the bifundamental hypermultiplets).
Therefore, the T-duality relation above leads to a duality relation between these two gauge theories in which the coupling constants are exchanged with the Coulomb branch parameters and vice versa.

It should be noted that the corresponding instanton partition functions can be obtained by suitable expansions of the topological string partition function $\mathcal{Z}_{N, M}$. Indeed, in [22], these two gauge theories have also been referred to as vertical and horizontal description, respectively, reflecting two possible choices of a preferred direction in the toric web diagram of $X_{N, M}$ for calculating $\mathcal{Z}_{N, M}$ using the (refined) topological vertex: the web diagram is shown in Fig. 1, with a generic labeling of the various line segments, which represent rational curves in $X_{N, M}$. Since each trivalent vertex contains a horizontal (green) leg and a vertical (red) leg, we can choose either of them as the preferred direction, which gives rise to a representation of $\mathcal{Z}_{N, M}$ as a series expansion in $e^{-h_{1, \ldots, M N}}$ or $e^{-v_{1, \ldots, M N}}$, respectively. At the particular region in the moduli space considered in [22], these in turn could readily be identified with the instanton expansions of the quiver gauge theories mentioned above.

However, inspecting Fig. 1, we see that all vertices also contain a diagonal (blue) leg, which can equally be chosen as the preferred direction. Hereafter, we refer to this as “diagonal description." In fact, in [36], diagonal expansion

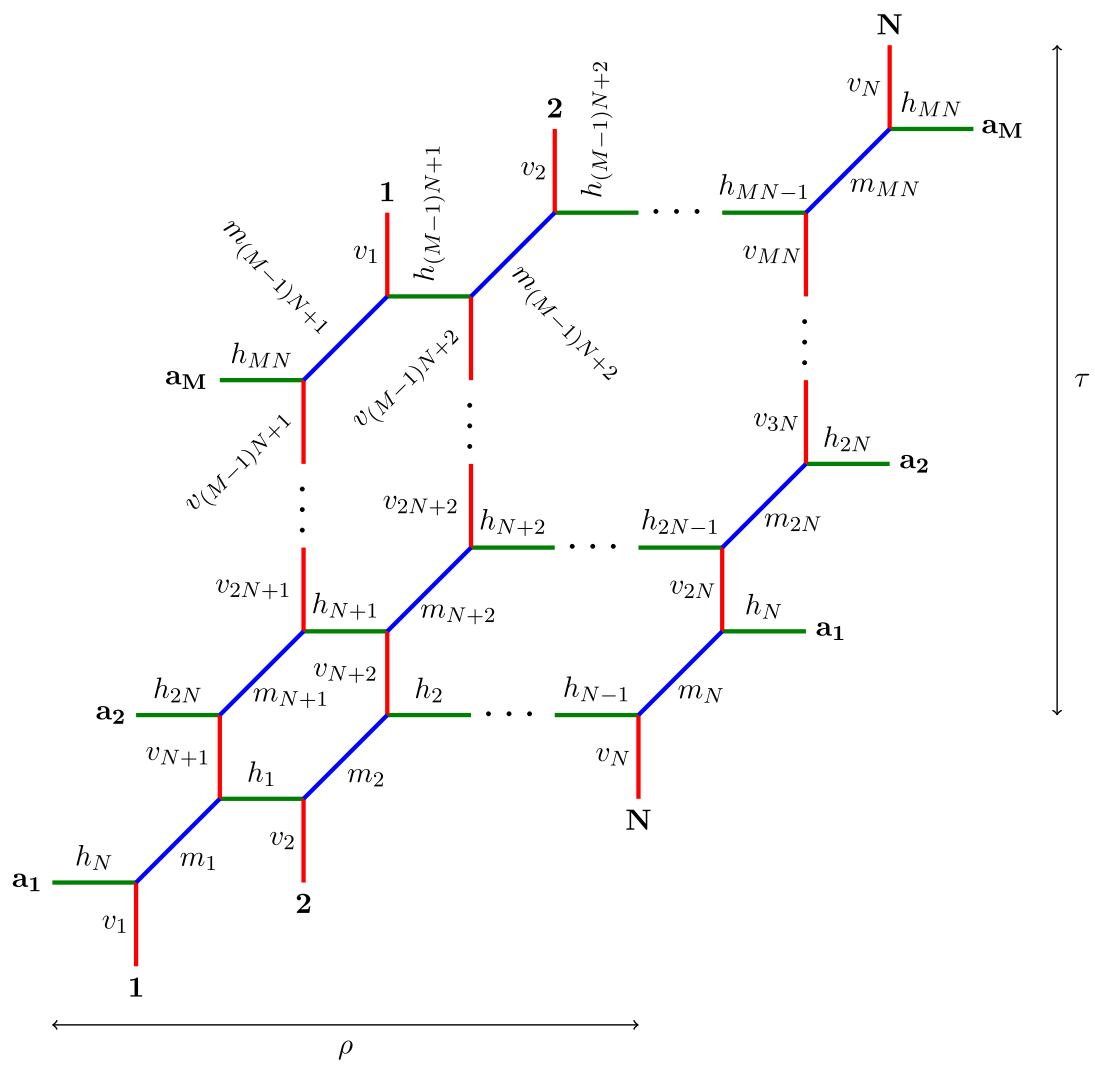

FIG. 1. The 5-brane web corresponding to $X_{N, M}$ with a generic parametrization of all line segments. Not all variables $\mathbf{h}=\left(h_{1}, \ldots, h_{M N}\right), \mathbf{v}=\left(v_{1}, \ldots, v_{M N}\right)$ and $\mathbf{m}=\left(m_{1}, \ldots, m_{M N}\right)$ are independent, but are subject to $2 N M-2$ consistency conditions. 
of $\mathcal{Z}_{N, M}$ in the form of a series in powers of $e^{-m_{1, \ldots, N M}}$ have recently been studied. While well-defined from the perspective of the topological string, it is an interesting question if this presentation of $\mathcal{Z}_{N, M}$ (in some region of the parameter space) can also be interpreted as the instanton partition function of a new (quiver) gauge theory that can be engineered from $X_{N, M}$. If this were so, it would extend the T-duality relation of LSTs to a triality. In this paper, we present strong evidence that this is indeed the case and that the diagonal description gives a (circular) quiver gauge theory with gauge group $[U(N M / k)]^{k}$ [where $k=\operatorname{gcd}(N, M)]$, which is the weak coupling limit of a new LST. Working at a generic point in the Kähler moduli space of $X_{N, M}$, we analyze in detail the horizontal, vertical and diagonal gauge theories, whose gauge group is

$G_{\mathrm{hor}}=[U(M)]^{N}, \quad G_{\mathrm{vert}}=[U(N)]^{M}, \quad G_{\mathrm{diag}}=\left[U\left(\frac{N M}{k}\right)\right]^{k}$,

respectively. We propose explicit parametrizations of the Kähler cone of $X_{N, M}$ which makes these three gauge symmetries manifest by allowing to extract the corresponding instanton partition functions $Z_{\mathrm{hor}}^{(N, M)}, Z_{\mathrm{vert}}^{(N, M)}$ and $Z_{\mathrm{diag}}^{(N, M)}$ from $\mathcal{Z}_{N, M}$.

The Alday-Gaiotto-Tachikawa (AGT) relation for fourdimensional $\mathcal{N}=2$ supersymmetric gauge theories [37,38] has been extended to five-dimensional gauge theories [39-41] and to five-dimensional quiver gauge theories [42]. The triality we newly proposed in this paper has interesting implications for this AGT relation. It is known that there is a relation between the five-dimensional $[U(N)]^{M}$ quiver gauge theory and an $W_{N}$ algebra [41]. By duality, there is also a relation between the same quiver gauge theory and an $W_{M}$ algebra [42]. The newly proposed triality implies a further relation between the same quiver gauge theory and an $W_{\frac{N M}{k}}$ algebra.

Finally, based on our previous work [36], we remark that in the extended Kähler moduli space of $X_{N, M}$ (extended through flop transformations) there are yet more LSTs. By analyzing the associated toric diagrams, it was shown in [11] that $X_{N, M} \sim X_{N^{\prime}, M^{\prime}}$ for $M N=M^{\prime} N^{\prime}$ and $\operatorname{gcd}(M, N)=k=\operatorname{gcd}\left(M^{\prime}, N^{\prime}\right)$; i.e., the two Calabi-Yau threefolds lie in the same extended moduli space and can be related by a combination of symmetry and flop transforms. For example, the Kähler cone of $X_{N^{\prime}, M^{\prime}}$ again affords different parametrizations, which suggest the appearance of a triad of quiver gauge theories, with gauge groups $\left[U\left(M^{\prime}\right)\right]^{N^{\prime}},\left[U\left(N^{\prime}\right)\right]^{M^{\prime}}$ and $\left[U\left(N^{\prime} M^{\prime} / k\right)\right]^{k}$, which are generically different from the theories mentioned above. These, along with other dual quiver gauge theories and associated $\mathrm{W}$-algebras, will be discussed in detail in the forthcoming publication [43].

This paper is organized as follows. In Sec. II, we discuss LSTs of type $(N, M)$ from the viewpoints of branes in
M-theory, $(p, q)$ 5-brane webs in type IIB theory, and dual Calabi-Yau threefolds $X_{N, M}$. We also discuss the Kähler cone of $X_{N, M}$ and the theory data of the dual gauge theories. In Sec. III, we analyze three different limits of the LST partition function leading to distinct (but dual) weakly coupled affine A-type quiver gauge theories. In Sec. IV, we make some general comments about the implication of triality for the W-algebras associated with these gauge theories. In Sec. V, we present our conclusions and directions for future work. In the Appendix, we relegate special situations of $(N, N)$ and $(n N, N)$ webs. We also elaborate the residual dualities that survive in the noncompact limit that the LSTs descend to superconformal field theories.

\section{LITTLE STRINGS, GAUGE THEORIES, AND BRANE WEBS}

In this section, we briefly review how six-dimensional LSTs with $\mathcal{N}=(2,0)$ and $\mathcal{N}=(1,0)$ supersymmetry and world volume $\mathbb{R}^{4} \times \mathbb{T}^{2}$ can be constructed from brane configurations in M-theory, along with their parameter space. These M5-brane configurations are U-dual to a class of $(p, q)$ 5-brane webs in type IIB string theory and can give rise to, in the limit of decoupling little strings, fourdimensional superconformal field theories (SCFTs) with eight supercharges in the infrared. We will also discuss in this section the gauge group and the coupling constants of the quiver gauge theories dual to LSTs on $\mathbb{R}^{4} \times \mathbb{T}^{2}$ and the Kähler cone of the associated Calabi-Yau threefold $X_{N, M}$ which is dual to the $(p, q)$ 5-brane web.

\section{A. Little string theories from M-theory}

In this subsection, we discuss how $\mathcal{N}=(1,0)$ LSTs arise from M-theory along with their parameter spaces. We begin with a brief review of the M-brane configurations which engineer LSTs. For more details on this construction, see $[22,25-27]$.

\section{M-brane configurations}

A stack of $N$ coincident M5-branes probing a transverse $\mathbb{R}^{5}$ space at low energies is described by a six-dimensional $\mathcal{N}=(2,0)$ SCFT of $A_{N-1}$ type [44-48]. We can move away from the conformal point by separating the M5branes along a $\mathbb{R}_{\text {trans }} \subset \mathbb{R}^{5}$ transverse to the branes: indeed, in this case massive states appear in the form of M2-branes stretched between the individual M5-branes. If $\mathbb{R}_{\text {trans }}$ is furthermore compactified to a circle $\mathbb{S}_{\text {trans }}^{1}$ of circumference $\rho$, the dynamics on the $N$ M5-branes, whether coincident or not, defines a maximally supersymmetric LST of $A$-type. The positions of the M5-branes on the transverse circle parametrize a $\left(\mathbb{S}^{1}\right)^{N} / S_{N}$ moduli subspace of the tensor branch of the $N$ M5-branes on the (partially) compactified space. The circumference $\rho$ sets a defining length scale of 
the LST, which appears since an M2-brane can wrap $\mathbb{S}_{\text {trans }}^{1}$ while ending on separate M5-branes, thus giving rise to a string configuration whose tension $T \sim \rho$ (in M theory unit) is proportional to $\rho$. If the $\mathbb{S}_{\text {trans }}^{1} \times \mathbb{R}^{4}$ transverse to the M5-branes is orbifolded by $\mathbb{Z}_{M}$ (i.e., replaced by $\left.\mathbb{S}_{\text {trans }} \times \mathbb{R}^{4} / \mathbb{Z}_{M}\right)$, we obtain a LST with $\mathcal{N}=(1,0)$ supersymmetry. The spectrum of the latter is consists of towers of tensor multiplets, vector multiplets, and hypermultiplets.

The configurations we are mostly concerned with in this work are obtained by compactifying not only the time direction on $\mathbb{S}_{0}^{1}$ (whose radius is chosen to be 1), but also a further direction parallel to the M5-branes on $\mathbb{S}_{\|}^{1}$ (with circumference $\tau$ ), such that the world volume of the M5-branes becomes $\mathbb{S}_{0}^{1} \times \mathbb{S}_{\|}^{1} \times \mathbb{R}^{4}$. As has been discussed in detail in [26], these M-brane configurations are U-dual to webs of $M$ parallel D5-branes intersecting $N$ parallel NS5-branes in type II string theory. Upon resolving the intersections of these branes, the web can be presented in the form shown in Fig. 1. This description in turn is dual to F-theory compactifications on a toric Calabi-Yau manifold $X_{N, M}$, whose toric web diagram takes the same form as Fig. 1. For more details on these dual descriptions, we refer the reader to $[26,27,29]$ and references therein.

\section{Little strings and their parameter space}

The brane web shown in Fig. 1 is dual to the Calabi-Yau threefold $X_{N, M}$ with sizes of the various line segments corresponding to the Kähler parameters of $X_{N, M}$ [49]. The $3 N M$ parameters labeling the web, as shown in Fig. 1, (hereafter we collectively denote them as $[\mathbf{h}, \mathbf{v}, \mathbf{m})]$ are not all independent. There are only $(N M+2)$ independent Kähler parameters (see [27]), as they are constrained by the condition that all horizontal, vertical and diagonal lines must be parallel (i.e., oriented along $(1,0),(0,1)$, and $(1,1)$, respectively). Considering a hexagon web in Fig. 1 consisting of two horizontal, two vertical, and two diagonal lines (see Fig. 2 with some generic labeling of the areas associated with these line segments), the condition that the horizontal, vertical and diagonal lines are pairwise parallel, leads to the following constraints

$$
h+m=h^{\prime}+m^{\prime} \quad \text { and } \quad v+m^{\prime}=m+v^{\prime} .
$$

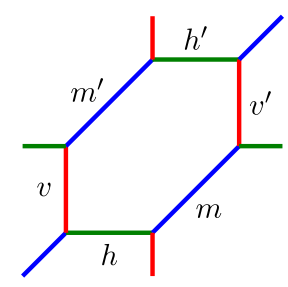

FIG. 2. Imposing consistency conditions on a hexagon appearing in Fig. 1.
Imposition of these conditions for every hexagon appearing in Fig. 1 leads to a system of linear equations whose solution contains $(M N+2)$ independent parameters (see [11] for more details). How to choose these independent variables is a priori not fixed and, as we shall see in following section, different choices allow us to explore different gauge theories engineered by the web diagrams.

We also remark that, in many works in the literature (see $[12,22,27,29,50])$, a nonmaximal set of parameters $\left(T_{1}, \ldots, T_{M}, t_{1}, \ldots, t_{N}, m\right)$ is often considered, which (with respect to the labeling of Fig. 1) is related to our parametrization as

$$
\begin{array}{ll}
T_{i}=m_{(i-1) N+s}+v_{i N+s}, & \forall s=1, \ldots, N \text { and } i=1, \ldots, M, \\
t_{a}=m_{a+r N}+h_{a+r N}, & \forall r=0, \ldots, M-1 \text { and } a=1, \ldots, N, \\
m=m_{k}, & \forall k=1, \ldots, N M .
\end{array}
$$

Indeed, the set of $(N+M+1)$ variables $\left(T_{1}, \ldots, T_{M}\right.$, $\left.t_{1}, \ldots, t_{N}, m\right)$ is a solution of the consistency conditions of the type (2.1).

The partition function of the LSTs engineered by the M-brane configurations corresponding to the web diagram in Fig. 1 is computed by the refined topological string partition function $\mathcal{Z}_{N, M}$ of the Calabi-Yau threefold $X_{N, M}$. This partition function depends on the Kähler parameters $(\mathbf{h}, \mathbf{v}, \mathbf{m})$ (subject to the consistency conditions mentioned above). In addition, it depends on the two refinement parameters $\epsilon_{1}$ and $\epsilon_{2}$ required by the refined topological string:

$$
\mathcal{Z}_{N, M}\left(\mathbf{h}, \mathbf{v}, \mathbf{m}, \epsilon_{1}, \epsilon_{2}\right)
$$

From the perspective of the gauge theories engineered by $X_{N, M}, \epsilon_{1,2}$ parametrize the $\Omega$ background (see $[30,51,52]$ ), suitable for regularizing the instanton contribution. Moreover, $\mathcal{Z}_{N, M}$ can be computed with the help of the refined topological vertex, which requires picking a preferred direction in the web diagram. Each choice of the preferred direction corresponds to a possibly distinct gauge theory partition function $[26,28,36]$.

\section{B. Dual gauge theories and series expansions}

\section{Theory data}

At low energies below the compactification scale, the LSTs associated with the Calabi-Yau threefolds $X_{N, M}$ discussed above are described by five-dimensional quiver gauge theories of $\hat{A}_{r-1}$ type with a unitary gauge group at each node $U(s)_{a}(a=1, \ldots, r)$. For a general low-energy description of M-theory compactified on a Calabi-Yau threefold, see [53-55]. The field content of these theories includes gauge vector multiplets $\left(\varphi_{a}, A_{a}\right)$, transforming in the adjoint of $U(s)_{a}$, and matter hypermultiplets $\left(H^{a}, \tilde{H}^{a}\right)$, transforming in the bifundamental representations of 
$U(s)_{a} \times U(s)_{a+1}$. From the perspective of the gauge theories, at a generic point of the Coulomb branch of the theory with gauge group $G$, the dynamics is described by the prepotential $\mathcal{F}$,

$$
\begin{aligned}
\mathcal{F}= & \frac{1}{2 !} t_{i j} \varphi^{i} \varphi^{j}+\frac{1}{3 !} c_{i j k}^{0} \varphi^{i} \varphi^{j} \varphi^{k} \\
& +\frac{1}{12}\left(\sum_{R}|R \cdot \varphi|^{3}-\sum_{f} \sum_{w \in W_{f}}\left|w \cdot \varphi+m_{f}\right|^{3}\right) .
\end{aligned}
$$

Here, $\varphi$ 's are local coordinates in an open patch of the Coulomb branch moduli space $\mathcal{M}$ and are the vacuum expectation values of the scalars in the vector multiplet with $i=1, \ldots, \operatorname{rank}(G) . R$ is the set of weights of the adjoint representation, $W_{f}$ are the sets of weights in the representation of the hypermultiplets with mass $m_{f}$ which for us is the bifundamental representation and the effective gauge couplings are given by,

$$
\left(g^{-2}\right)_{i j}=\partial_{i} \partial_{j} \mathcal{F}=t_{i j}+\cdots .
$$

The five-dimensional Lagrangian contains the ChernSimons term,

$$
\begin{aligned}
\mathcal{L}= & \frac{1}{24 \pi^{2}} c_{i j k} A^{i} \\
& \wedge F^{j} \wedge F^{k} \\
\text { where } \quad c_{i j k} & =\frac{\partial^{3} \mathcal{F}}{\partial \varphi_{i} \partial \varphi_{j} \partial \varphi_{k}}=c_{i j k}^{0}+\cdots,
\end{aligned}
$$

and $F^{i}$ is the field strength two-form associated with gauge potential one-form $A^{i}$. The presence of this term spoils the gauge invariance of the path integral at quantum level unless the coefficients $c_{i j k}$ are integrally quantized (in suitable units) and therefore cannot change under continuous symmetry transformations of the theory.

Recall that, if the five-dimensional theory were obtained by compactification of M-theory on a Calabi-Yau threefold, then the prepotential of the theory is related to the triple intersection of the divisors. For instance, consider a fivedimensional $S U(N)$ gauge theory obtained from a CalabiYau threefold $Y_{N}$ which is an $A_{N-1}$ singularity fibered over $\mathbb{P}^{1}$. Resolving the $A_{N-1}$ singularity gives a chain of $(N-1)$ $\mathbb{P}^{1}$ 's in the fiber. The compact divisors of $Y_{N}$ are the 4-cycles $\left\{D_{1}, D_{2}, \ldots, D_{N-1}\right\}$, which are the $i$-th fiber $\mathbb{P}^{1}$ fibered over the base $\mathbb{P}^{1}$. Each of the divisors $D_{i}$ is a Hirzebruch surface. The size of the $\mathbb{P}^{1}$ 's in the fiber is $t_{i}=\left(\varphi_{i+1}-\varphi_{i}\right)(i=1, \ldots, N)$ where $\varphi_{i}$ 's are the scalars introduced above. The cubic part of the prepotential, which gives the Chern-Simons coefficient (2.5), of the fivedimensional theory is then given by [56]

$$
\left.\mathcal{F}\right|_{\text {cubic }}=\frac{1}{6}\left(\sum_{i, j=1}^{N-1}\left(A^{-1}\right)^{i j} t_{i} D_{j}\right)^{3},
$$

where $\left(A^{-1}\right)^{i j}$ is the inverse Cartan matrix of $A_{N-1}$ and $D_{i} D_{j} D_{k}$ are the triple intersection numbers of the divisors in $Y_{N}$. From this, we can see that the coefficients $c_{i j k}$ in Eq. (2.5) depend on the triple intersection numbers of the divisors in the Calabi-Yau threefold $Y_{N}$. When the fivedimensional theory is considered on $\mathbb{R}^{4} \times \mathbb{S}^{1}$, the M2branes wrapping the holomorphic curves in $Y_{N}$ and the $\mathbb{S}^{1}$ (with momentum along the $\mathbb{S}^{1}$ ) contribute to the prepotential as well. In the type IIA string description, these are the bound-states of D2-branes and the D0-branes. The triple intersection numbers now get contributions from these bound-states such that

$$
D_{i} D_{j} D_{k} \mapsto D_{i} D_{j} D_{k}+\sum_{\beta \in H_{2}\left(Y_{N}, \mathbb{Z}\right)} N_{i j k}^{\beta} e^{-\int_{\beta} \omega},
$$

where $N_{i j k}^{\beta}$ is the contribution from the holomorphic curves in the class $\beta$ which intersect the divisors $D_{i}, D_{j}$ and $D_{k}$. These contributions are precisely captured by the genuszero topological string amplitude. The full partition function of the gauge theory on $\mathbb{R}^{4} \times \mathbb{S}^{1}$ is thus given by the topological string partition function of the corresponding Calabi-Yau threefold used to engineer the gauge theory itself $[57,58]$.

We can relate this gauge theory description to the CalabiYau description discussed in the previous section: the Kähler parameters of $X_{N, M}$ take the role of either the gauge coupling constants, Coulomb branch parameters or hypermultiplet masses. As already alluded to above, the precise correspondence is not unique; i.e., the $(N M+2)$ independent Kähler parameters can be assigned in different ways to these three sets of gauge theory parameters. This means that in general different gauge theories can be associated with a single $X_{N, M}$ which, however, are dual to each other. To clarify more precisely what we mean by "different but dual," we need to provide more details on the characteristic quantities that are necessary to distinguish two gauge theories engineered from the same $X_{N, M}$. They are as follows:

(i) gauge group $G$

The gauge theories discussed above that are associated with $X_{N, M}$ are of $\hat{A}$ quiver type with gauge group

$$
G=[U(s)]^{r}, \quad \text { with } \quad r s=N M .
$$

Here the condition $r s=N M$ can be understood from the fact that these gauge theories are lowenergy limits of LSTs of A-type, which can be obtained as a particular decoupling limits of type IIB string theory: conservation of D-brane charges in the latter imposes that the rank of the gauge group, $|G|$, is the same for all theories associated with $X_{N, M}$. 
(ii) gauge coupling constants

To each of the $r$ factors of $U(s)$ in the gauge group $G$ in (2.8), we can associate an independent inverse coupling constant $g_{i}^{-2}$. The coupling constants can be varied continuously and the weak coupling limit is $g_{i}^{-2} \rightarrow \infty$ for $i=1, \ldots, r$.

(iii) moduli space

The $(N M+2-r)$ remaining independent Kähler parameters of $X_{N, M}$ parametrize the hypermultiplet masses and Coulomb branch parameters of the gauge theory. The latter can be varied continuously and we assume to always work at a point in the moduli space where the latter are positive definite.

(iv) Chern-Simons terms

As we just discussed, the five-dimensional gauge theories contain Chern-Simons couplings for the vector multiplet gauge fields Eq. (2.5). The coefficients $c_{i j k}$ in this term are given by the triple intersection number of the Calabi-Yau threefold. They appear in the topological string partition function of the corresponding Calabi-Yau threefolds [59] (see also [60]). Recall that in general the topological string partition function of a CalabiYau threefold $X$ is given by

$$
Z_{X}=\exp (F),
$$

where $F=\sum_{g=0}^{\infty} \lambda^{2 g-2} F_{g}$ is the free energy given by the topological string coupling $\lambda$ and the genus- $g$ topological string amplitudes $F_{g}$ which are given by integrating topological string measure over the moduli space of genus- $g$ curves. If we denote the Kähler class of the Calabi-Yau threefold $X$ by $\omega$ $[61,62]$,

$$
\begin{aligned}
F= & \frac{1}{\lambda^{2}} \int_{X} \frac{1}{3 !} \omega \wedge \omega \wedge \omega \\
& -\int_{X} \frac{c_{2}(X)}{24} \wedge \omega+\frac{\chi(X)}{2} \ln M\left(e^{i \lambda}\right) \\
& +\sum_{0 \neq \beta \in H_{2}(X, \mathbb{Z})} \sum_{g \geq 0} n^{g}(\beta) \\
& \times \sum_{n=1}^{\infty} \frac{1}{n}\left(2 \sin \left(\frac{n \lambda}{2}\right)\right)^{2 g-2} e^{-n \int_{\beta} \omega},
\end{aligned}
$$

where $c_{2}(X)$ is the second Chern class of the tangent bundle of $X, \chi(X)$ is the Euler characteristic of $X$ and $M(q)$ is the MacMahon function,

$$
M(q)=\prod_{k=1}^{\infty}\left(1-q^{k}\right)^{-k} .
$$

The $n^{g}(\beta)$ are the genus- $g$ Gopakumar-Vafa invariants $[61,62]$ of the curve class $\beta$ and are conjectured to be integers since they are related to the dimensions of the various homology groups of the moduli space of D2-branes wrapping the holomorphic curve in class $\beta$. The genus-zero contribution to the free energy contains the classical contribution $\int_{X} \omega \wedge$ $\omega \wedge \omega$ which gives the triple intersection numbers $\left[\omega=\sum_{i=1}^{\operatorname{dim} H^{1,1}(X, \mathbb{C})} x^{i} \omega_{i}\right.$ where $\left.\omega_{i} \in H^{1,1}(X, \mathbb{C})\right]$,

$$
\begin{aligned}
\int_{X} \omega \wedge \omega \wedge \omega & =c_{i j k} x^{i} x^{j} x^{k} \\
\text { where } \quad c_{i j k} & =\int_{X} \omega_{i} \wedge \omega_{j} \wedge \omega_{k} .
\end{aligned}
$$

From this, we see that the triple intersection numbers and hence the Chern-Simons coefficients appear in the definition of $F_{0}$ (the genus-zero amplitude) and hence in the partition function. However, this is not the only place the Chern-Simons terms appear. Since the geometry of the Calabi-Yau threefold is related to the triple intersection numbers, the GopakumarVafa invariants $n^{g}(\beta)$ change as the triple intersection numbers change. One can see this very clearly when the topological string partition function is expressed in the form of Nekrasov's instanton partition function.

In the case of $S U(N)$ gauge theory we discussed above, the Chern-Simons coefficient can take values from $-N$ to $+N$, where theories of opposite sign Chern-Simons coefficients are related by chargeparity conjugation. So, there are $(N+1)$ distinct gauge theories.This is reflected in the geometry as well since the fibration of the $A_{N-1}$ singularity over $\mathbb{P}^{1}$ is not unique either and there are actually $(N+1)$ distinct fibrations corresponding to distinct fivedimensional gauge theories. We denote by $Y_{N, k}$ the Calabi-Yau threefolds which engineer $S U(N)$ gauge theory with Chern-Simons coefficient $k$.

The topological string partition function of $Y_{N, k}$ were calculated in [58] (for the refined case, see [63]) and it was found that partition functions for different $k$ were related to each other. Define the topological string partition function of $Y_{N, k}$ as

$$
Z_{Y_{N, k}}=\sum_{\nu_{1} \cdots \nu_{N}} Q_{B}^{\left|\nu_{1}\right|+\cdots+\left|\nu_{N}\right|} Z_{\nu_{1} \cdots \nu_{N}}^{k}(\mathbf{t}, \epsilon)
$$

where $-\ln Q_{B}$ is the Kähler parameter of the base $\mathbb{P}^{1}$. Then,

$$
Z_{\vec{\nu}}^{k}(\mathbf{t}, \epsilon)=f_{\vec{\nu}}^{k}(\mathbf{t}, \epsilon) Z_{\vec{\nu}}^{0}(\mathbf{t}, \epsilon)
$$

where the $k$-dependent part reads 


$$
\begin{aligned}
f_{\vec{\nu}}^{k}(\mathbf{t}, \epsilon) & =\left(\prod_{i=1}^{\left\lfloor\frac{N+k-1}{2}\right\rfloor} Q_{t_{i}}^{(N+k-2 i)\left(\left|\nu_{1}\right|+\cdots+\left|\nu_{i}\right|\right)}\right. \\
\times & \left.\prod_{i=\left\lfloor\frac{N-k+1}{2}\right\rfloor}^{N-1} Q_{t_{i}}^{-(N+k-2 i)\left(\left|\nu_{i+1}\right|+\cdots+\left|\nu_{N}\right|\right)}\right) q^{\frac{1}{2} k \sum_{i=1}^{N} k\left(\nu_{i}\right)},
\end{aligned}
$$

where $\kappa(\nu)=\sum_{(i, j) \in \nu}(j-i)$. Thus, we see that the effect of the Chern-Simons coefficient is to modify the prefactor once the topological string partition function is expressed in the form of the Nekrasov partition function.

The nonperturbative gauge theory partition function for a theory with the gauge group $G$ in Eq. (2.8) can be obtained by writing $\mathcal{Z}_{N, M}$ as a series expansion in powers of $\exp \left[-1 / g_{a}^{2}\right]$. In practice, we will identify sets of parameters, the decoupling parameters $d_{a}$, such that in the limit $d_{a} \mapsto \infty$ the $(N, M)$ web breaks up into pieces whose partition function can be identified with the perturbative part of gauge theory partition function. The parameters $d_{a}$ are related to the gauge theory coupling constants $\frac{1}{g_{a}^{2}}$ such that

$$
d_{a} \mapsto \infty \Rightarrow \frac{1}{g_{a}^{2}} \mapsto \infty
$$

The $d_{a}$ 's differ from $\frac{1}{g_{a}^{2}}$ by a combination of Coulomb branch parameters and masses of the bifundamental hypermultiplets. Thus, we are naturally led to studying different types of series expansions of Eq. (2.3), as we shall do in the following.

\section{Series expansions and building blocks}

The topological string partition function $\mathcal{Z}_{N, M}$ appears naturally in the form of an infinite series when computed using the refined topological vertex. Indeed, gluing the trivalent vertices together according to the web diagram of $X_{N, M}$ requires to choose a preferred direction common to all vertices. Thus, the preferred direction is a feature of the entire web diagram and not only of an individual vertex. A given brane web, however, may allow for several different choices, which lead to different (but equivalent) representations of the topological string partition function. For web diagrams of the form shown in Fig. 1, we have three different choices of the preferred direction, namely, horizontal along $(1,0)$, vertical along $(0,1)$ and diagonal along $(1,1)$. For each of the three, the web diagram can be cut into strips as shown in Fig. 3, which represent the basic building blocks for computing $\mathcal{Z}_{N, M}$.

Here, the external legs of each of the strips are labeled in terms of two sets of integer partitions $\left\{\alpha_{1, \ldots, L}\right\}$ and $\left\{\beta_{1, \ldots, L}\right\}$ (where $L=M$ or $L=N$ or $L=\frac{N M}{k}$ for (a), (b) or (c), respectively, and $\beta^{t}$ denotes the transposed partition of $\beta$ ).

Since the three strips can be transformed into one another with the help of an $S L(2, \mathbb{Z})$-symmetry (under which the partition function is invariant), they can all be computed in the same fashion (using the refined topological vertex). Indeed, the common generic expression has been calculated explicitly in [36] for a generic parametrization. In order to find the strips in Fig. 3, we simply need to adapt the Kähler parameters accordingly. To be precise, in [36], the so-called

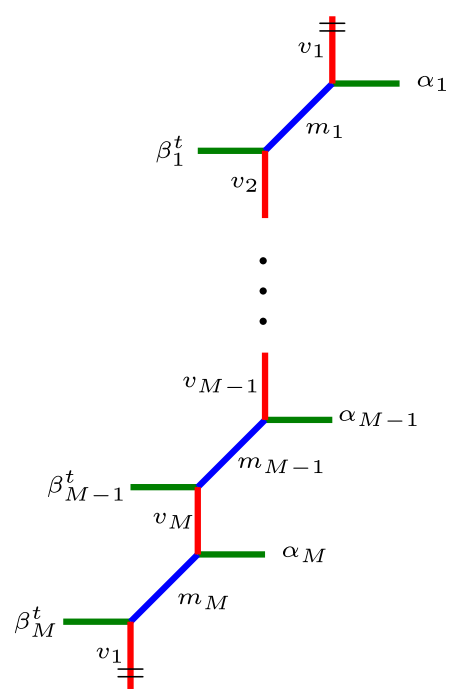

(a)

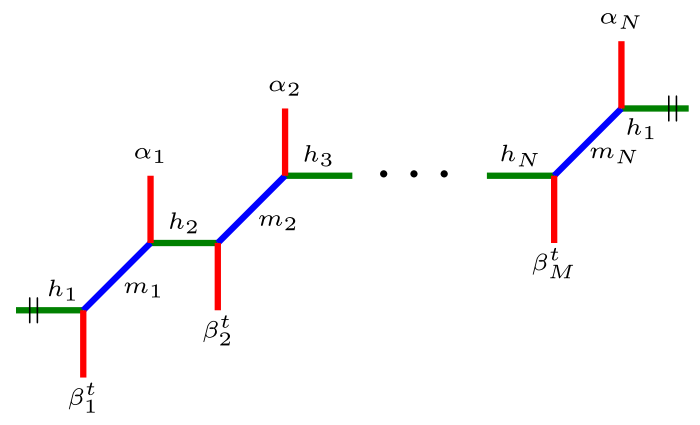

(b)

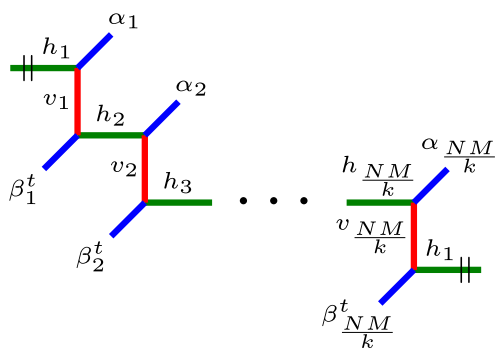

(c)

FIG. 3. Basic building blocks for decomposition of the web diagram Fig. 1: (a) strip of length $M$ representing the building block suitable for horizontal preferred direction (b) strip of length $N$ representing the building block suitable for vertical preferred direction (c) strip of length $\frac{N M}{k}$ [with $\left.k=\operatorname{gcd}(N, M)\right]$ representing the building block suitable for diagonal preferred direction. 
"staircase diagram" shown in Fig. 4 was considered, which is parametrized by $\left(\hat{a}_{1, \ldots, L}, \hat{b}_{1, \ldots, L}, S\right)$ (where $\left.\sum_{i=1}^{L}\left(\hat{a}_{i}-\hat{b}_{i}\right)=0\right)$. The latter can directly be adapted to fit each of the three strips (a), (b), and (c) in Fig. 3. Specifically, for $i=1, \ldots, L$, we have the following correspondence

\begin{tabular}{lccc}
\hline & Strip (a) & Strip (b) & Strip (c) \\
\hline$\hat{a}_{i}$ & $v_{i+1}+m_{i}$ & $h_{i}+m_{i}$ & $v_{i}+h_{i+1}$ \\
$\hat{b}_{i}$ & $v_{i}+m_{i}$ & $h_{i}+m_{i-1}$ & $h_{i}+v_{i}$ \\
$S$ & $v_{1}$ & $m_{N}$ & $h_{1}$ \\
$L$ & $M$ & $N$ & $\frac{N M}{k}$ \\
\hline
\end{tabular}

where we identified $h_{L+1}=h_{1}, v_{L+1}=v_{1}$ and $m_{0}=m_{L}$. Defining $q=e^{2 \pi i \epsilon_{1}}, t=e^{-2 \pi i \epsilon_{2}}$ and

$$
Q_{a_{i}}=e^{-\hat{a}_{i}}, \quad Q_{b_{i}}=e^{-\hat{b}_{i}}, \quad Q_{S}=e^{-S},
$$

as well as the following quantities

$$
\begin{aligned}
& \hat{Q}_{i, j}=Q_{S} \prod_{r=1}^{i}\left(Q_{a_{r}} Q_{b_{r}}^{-1}\right) \prod_{k=1}^{j-1} Q_{a_{i-k}}, \\
& \bar{Q}_{i, j}= \begin{cases}1 & \text { if } j=L \\
\prod_{k=1}^{j} Q_{a_{i-k}} & \text { if } j \neq L\end{cases} \\
& \dot{Q}_{i, j}=\prod_{k=1}^{j} Q_{b_{i+k}} .
\end{aligned}
$$

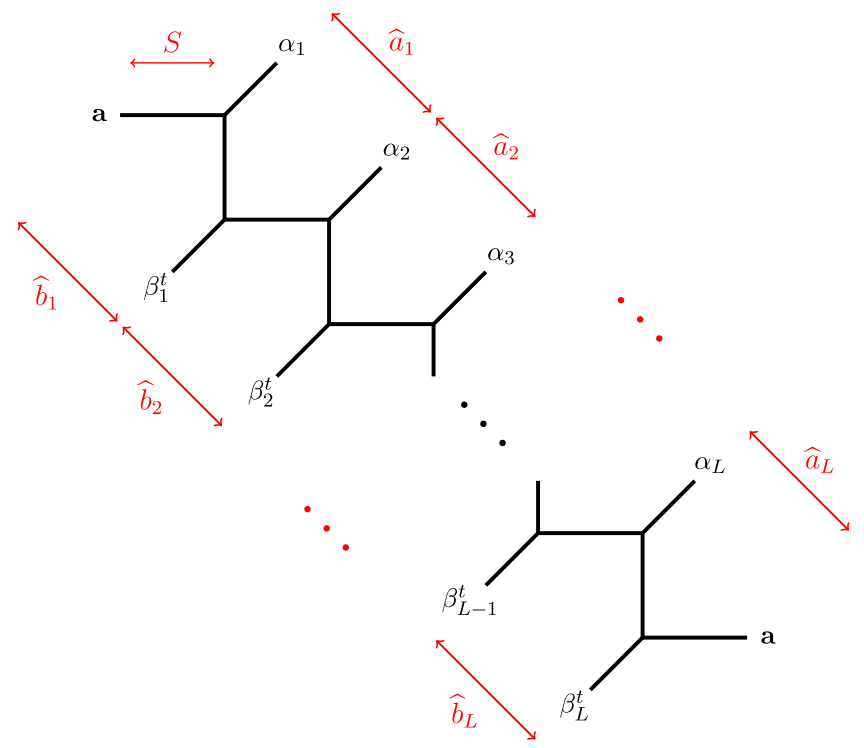

FIG. 4. Generic strip of length $L$ with a labeling of the various parameters suitable for the computation of the building block in Eq. (2.18). The external legs are labeled by integer partition $\left\{\alpha_{1, \ldots, L}\right\}$ and $\left\{\beta_{1}, \ldots, \beta_{L}\right\}$ (where $\beta_{i}^{t}$ denotes the transposed partition).

We can write the generic building block associated with the strip in Fig. 4 in the following fashion [36] (see also $[26,29]$ for earlier results working in the particular region (2.2) of the moduli space of $X_{N, M}$ )

$$
W_{\beta_{1} \ldots \beta_{L}}^{\alpha_{1} \ldots \alpha_{L}}=W_{L}(\varnothing) \cdot \hat{Z} \cdot \prod_{i, j=1}^{L} \frac{\mathcal{J}_{\alpha_{i} \beta_{j}}\left(\hat{Q}_{i, i-j} ; q, t\right) \mathcal{J}_{\beta_{j} \alpha_{i}}\left(\left(\hat{Q}_{i, i-j}\right)^{-1} Q_{\rho} ; q, t\right)}{\mathcal{J}_{\alpha_{i} \alpha_{j}}\left(\bar{Q}_{i, i-j} \sqrt{q / t} ; q, t\right) \mathcal{J}_{\beta_{j} \beta_{i}}\left(\dot{Q}_{i, j-i} \sqrt{t / q} ; q, t\right)},
$$

where the prefactors are given by (with $Q_{\rho}=\prod_{i=1}^{L} Q_{a_{i}}$ )

$$
\begin{aligned}
W_{L}(\varnothing) & =\prod_{i, j=1}^{L} \prod_{k, r, s=1}^{\infty} \frac{\left(1-\hat{Q}_{i, j} Q_{\rho}^{k-1} q^{r-\frac{1}{2}} t^{s-\frac{1}{2}}\right)\left(1-\hat{Q}_{i, j}^{-1} Q_{\rho}^{k} q^{s-\frac{1}{2}} t^{r-\frac{1}{2}}\right)}{\left(1-\bar{Q}_{i, j} Q_{\rho}^{k-1} q^{r} t^{s-1}\right)\left(1-\dot{Q}_{i, j} Q_{\rho}^{k-1} q^{s-1} t^{r}\right)}, \\
\hat{Z} & =\prod_{i=1}^{L} t^{\frac{\left\|\alpha_{k}\right\|^{2}}{2}} q^{\frac{\left\|\alpha_{k}^{t}\right\|^{2}}{2}} \tilde{Z}_{\alpha_{k}}(q, t) \tilde{Z}_{\alpha_{k}^{t}}(t, q), \quad \tilde{Z}_{\nu}(t, q)=\prod_{(i, j) \in \nu}\left(1-t^{\nu_{j}^{t}-i+1} q^{\nu_{i}-j}\right)^{-1},
\end{aligned}
$$

and the function $\mathcal{J}_{\mu \nu}$ (for two partitions $\mu$ and $\nu$ ) is defined as

$$
\begin{aligned}
\mathcal{J}_{\mu \nu}(x ; t, q)= & \prod_{k=1}^{\infty} J_{\mu \nu}\left(Q_{\rho}^{k-1} x ; t, q\right), \\
J_{\mu \nu}(x ; t, q)= & \prod_{(i, j) \in \mu}\left(1-x t^{t_{j}^{t}-i+\frac{1}{2}} q^{\mu_{i}-j+\frac{1}{2}}\right) \\
& \times \prod_{(i, j) \in \nu}\left(1-x t^{-\mu_{j}^{t}+i-\frac{1}{2}} q^{-\nu_{i}+j-\frac{1}{2}}\right) .
\end{aligned}
$$

Gluing several of strips together (see [36] for more details), we get

$$
\mathcal{Z}_{N, M}=\sum_{\boldsymbol{\alpha}}\left(\prod_{i=1, j=1}^{M, N} e^{-u_{i j}\left|\alpha_{j}^{i}\right|}\right) \prod_{j=1}^{N} W_{\alpha_{j+1}^{1} \cdots \alpha_{j+1}^{M}}^{\alpha_{j}^{1} \cdots \alpha_{j}^{M}}
$$

where $e^{-u_{i j}}$ are the parameters used to glue the strips together and we are using the fact that the compactification of the web gives $\alpha_{N+1}^{i}=\alpha_{1}^{i}$. We can obtain three different series representations of $\mathcal{Z}_{N, M}$ which we write in the following form, 


$$
\begin{aligned}
\mathcal{Z}_{N, M}\left(\mathbf{h}, \mathbf{v}, \mathbf{m}, \epsilon_{1,2}\right) & =Z_{p}(\mathbf{v}, \mathbf{m}) \sum_{\vec{k}} e^{-\vec{k} \cdot \mathbf{h}} Z_{\vec{k}}(\mathbf{v}, \mathbf{m})=Z_{\text {hor }}^{(N, M)} \\
& =Z_{p}(\mathbf{h}, \mathbf{m}) \sum_{\vec{k}} e^{-\vec{k} \cdot \mathbf{v}} Z_{\vec{k}}(\mathbf{h}, \mathbf{m})=Z_{\text {vert }}^{(N, M)} \\
& =Z_{p}(\mathbf{h}, \mathbf{v}) \sum_{\vec{k}} e^{-\vec{k} \cdot \mathbf{m}} Z_{\vec{k}}(\mathbf{h}, \mathbf{v})=Z_{\text {diag }}^{(N, M)},
\end{aligned}
$$

where $Z_{p}$ denotes the perturbative part in each of the three descriptions. The first two of these expansions $\left(Z_{\mathrm{hor}}^{(N, M)}\right.$ and $Z_{\mathrm{vert}}^{(N, M)}$ ) have been extensively studied in the literature $[26,29]$ at a point in the Kähler moduli space of $X_{N, M}$ where $m_{i}=m_{j}$ (for $i, j=1, \ldots, N M$ ). The diagonal expansion $Z_{\text {diag }}^{(N, M)}$ has recently [36] been used to prove the duality [11] $X_{N, M} \sim X_{N^{\prime}, M^{\prime}} \quad$ [with $\quad N^{\prime} M^{\prime}=N M$ and $\left.\operatorname{gcd}(N, M)=k=\operatorname{gcd}\left(N^{\prime}, M^{\prime}\right)\right]$ explicitly for $k=1$ and give further evidence for $k>1$. However, its gauge theory interpretation has not been studied so far.

The main proposition of this paper is to interpret the three different expansions in (2.21) as instanton expansions in field theory. Therefore, we associate a triality of gauge theories to a given Calabi-Yau threefold $X_{N, M}$. However, identifying $Z_{\mathrm{hor}}^{(N, M)}, Z_{\mathrm{vert}}^{(N, M)}$ or $Z_{\mathrm{diag}}^{(N, M)}$ in Eq. (2.21) with the instanton series of a gauge theory is, however, a priori delicate: indeed, due to the consistency conditions discussed in Sec. II A 2, in general, the parameters $(\mathbf{h}, \mathbf{v}, \mathbf{m})$ are not independent of one another and therefore it is not clear whether (2.21) can be interpreted as (consistent) power series expansions in a set of independent parameters that can be identified with the gauge coupling parameters of a gauge theory. For this to be the case, we need to show that there exists a codimension $n$ region in the Kähler moduli space of $X_{N, M}$ in which either all $\mathbf{h}$ or $\mathbf{v}$ or $\mathbf{m}$ become infinite, while all the remaining parameters are finite. From the gauge theory perspective, this region corresponds to the weak-coupling regime and in a finite neighborhood of it either $Z_{\mathrm{hor}}^{(N, M)}$ or $Z_{\mathrm{vert}}^{(N, M)}$ or $Z_{\mathrm{diag}}^{(N, M)}$ in (2.21), respectively, are well defined power series expansions of $\mathcal{Z}_{N, M}$ that can be identified as an instanton expansion.

\section{Kähler cone}

To give a more geometric picture of the situation, we first recall that the Kähler moduli space of $X_{N, M}$ takes the form of a cone, as shown in Fig. 5. Denoting the Kähler form of $X_{N, M}$ by $\omega$, the interior of this cone is parametrized by

$$
\begin{aligned}
& \int_{X_{N, M}} \omega \wedge \omega \wedge \omega>0, \text { and } \int_{P_{a}} \omega \wedge \omega>0, \\
& \text { and } \int_{\Sigma_{i}} \omega>0,
\end{aligned}
$$

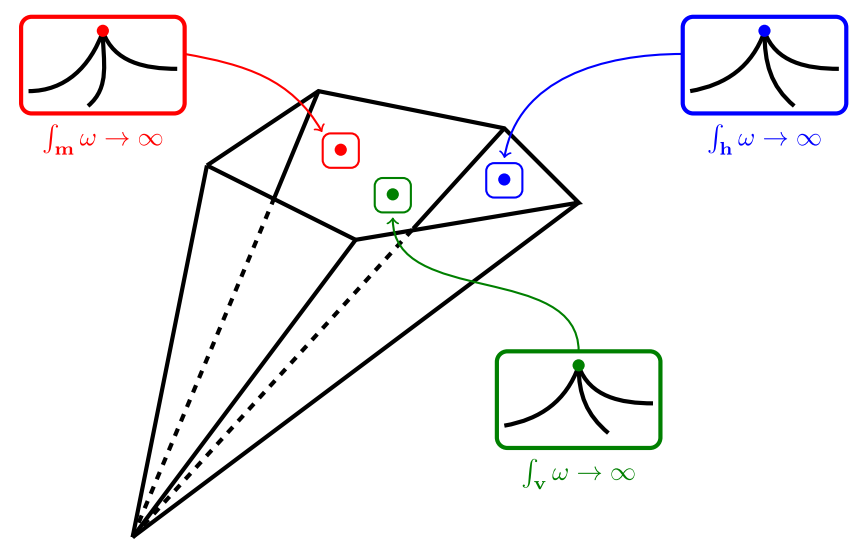

FIG. 5. Different regions in the Kähler cone of $X_{N, M}$.

where $P_{a}$ are two-complex-dimensional submanifolds and $\Sigma_{i} \in\{\mathbf{h}, \mathbf{v}, \mathbf{m}\}$ are holomorphic curves in $X_{N, M} .{ }^{1}$ The walls of the cone are given by

$$
\begin{aligned}
& \int_{X_{N, M}} \omega \wedge \omega \wedge \omega=0, \quad \text { and } \quad \int_{P_{a}} \omega \wedge \omega=0, \\
& \text { and } \quad \int_{\Sigma_{i}} \omega=0,
\end{aligned}
$$

and, in particular, include loci in which any of the parameters $(\mathbf{h}, \mathbf{v}, \mathbf{m})$ vanish. In these loci, the CalabiYau $X_{N, M}$ develops a singularity.

The weak coupling regions (from the point of view of the gauge theories engineered by $X_{N, M}$ ) proposed above are shown as the three colored areas in Fig. 5. They are characterized by (for $i=1, \ldots, M N$ )

$$
\begin{aligned}
& \text { - red: } \int_{m_{i}} \omega \rightarrow \infty, \text { and } \int_{h_{i}, v_{i}} \omega=\text { finite, } \\
& \text { - blue: } \int_{h_{i}} \omega \rightarrow \infty, \text { and } \int_{m_{i}, v_{i}} \omega=\text { finite, } \\
& \text { - green: } \int_{v_{i}} \omega \rightarrow \infty, \quad \text { and } \quad \int_{v_{i}, m_{i}} \omega=\text { finite. }
\end{aligned}
$$

respectively. Notice that the codimension of these regions are in general not the same. In the following section, we will give evidence that such three regions indeed exist in the interior of the Kähler cone of $X_{N, M}$ for generic $(N, M)$.

\section{Type IIB 5-brane web interpretation}

The triality of gauge theories alluded to in the previous subsubsection can also be argued for from the point of view of the $(p, q)$-brane web, which is dual to $X_{N, M}$. Indeed, the web contains D5-branes, NS5-branes and $(1,1)$ branes,

\footnotetext{
${ }^{1} \mathrm{By}$ abuse of notation, we use the same name for the curve as well as its area.
} 
associated with the horizontal, vertical and diagonal line segments in Fig. 1, respectively. The $S L(2, \mathbb{Z})$ symmetry of type IIB string theory acts in the form of rotations on the web and allows us to exchange the roles of these branes, respectively. This not only gives rise to a duality between the horizontal and vertical description in the notation above (which has been discussed in the literature at various places $[22,26,27])$, the S-duality also allows us to convert $(1,1)$ branes to D5-branes, thus providing another nonlocally dual description [i.e., the diagonal expansion in (2.21)].

With each brane web we can associate the gauge theory that lives on the world volume of the D5-branes. Since different line segments (from the perspective of the original $(p, q)$-brane web) play the role of the D5-branes in the various $S$-duality frames, we therefore also expect that the above $S L(2, \mathbb{Z})$ symmetry allows us to relate different gauge theories. As already mentioned above (see Sec. II A 1), while specific details of the theories (e.g., the precise form of the gauge group) are different, they share certain quantities in common (e.g., the rank of the gauge group).

Specifically, in the $(p, q)$ 5-brane web shown in Fig. 1 with $M$ horizontal D5-branes and $N$ NS5-branes intersecting them vertically, the theory on the former is a $G_{\text {hor }}=$ $[U(M)]^{N}$ quiver gauge theory of $A_{N-1}$ type, with bifundamental matter arising at the intersections. Furthermore, for nonzero separation between the branes, the gauge group is broken to $U(1)^{N M}$. Using the S-duality of the type IIB string theory, we can map the above $(p, q)$ 5-brane web to the S-dual web in which the identification of D5-branes and NS5-branes get exchanged so that we have $N$ D5-branes in the background of $M$ NS5-branes. This configuration then gives rise to another five-dimensional $G_{\text {vert }}=[U(N)]^{M}$ quiver gauge theory of $A_{M-1}$ type. As we shall discuss below, a similar duality frame allows us to associate a gauge theory with gauge group $G_{\text {diag }}=[U(N M / k)]^{k}[$ for $k=\operatorname{gcd}(N, M)]$ with the diagonal line segments.

\section{WEAK COUPLING LIMIT}

In the previous section, we have seen that a crucial step in associating a quiver gauge theory with a given CalabiYau manifold is to identify a region in the Kähler moduli space of the latter that can be identified with the weak coupling regime. In this section, we will analyze this weak coupling limit in more detail to provide evidence for the triality of gauge theories suggested by (2.21): we will first consider two examples in detail [namely, $(N, M)=(2,2)$ and $(3,2)]$ and generalize to generic $(N, M)$ later on.

\section{A. Configuration $(N, M)=(2,2)$}

As remarked above, the key aspect of interpreting $Z_{\mathrm{hor}}^{(N, M)}, Z_{\mathrm{vert}}^{(N, M)}$ or $Z_{\mathrm{diag}}^{(N, M)}$ in $(2.21)$ as instanton gauge theory partition functions, is to find a region in the Kähler moduli space of $X_{N, M}$ in which either all $\mathbf{h}$ or all $\mathbf{v}$ or all $\mathbf{m}$ become infinitely large, while the remaining parameters remain finite. In order to find such a region in the moduli space, we require a particular basis for the Kähler parameters, which provides a solution for the consistency conditions discussed in Sec. II A 2. While such a basis is very involved for generic $(N, M)$ (see Sec. III C for a proposal in the most general case), we first consider as a simple example the configuration $(N, M)=(2,2)$ (with $k=\operatorname{gcd}(N, M)=2$ ), to illustrate the point. In this case, three different parametrizations (suitable for the horizontal, vertical and diagonal gauge theory) along with a graphical interpretation of the weak coupling limit are shown in Table I. The three different expansions (and, in particular, the weak coupling limit) can be interpreted as follows:

(i) horizontal expansion in the basis $\left(\rho, \hat{b}_{1} ; \hat{c}_{1}, \hat{c}_{2}, \tau ; E\right)$ Upon taking the limit

$$
\rho-\hat{b}_{1} \rightarrow \infty, \quad \text { and } \quad \hat{b}_{1} \rightarrow \infty
$$

all horizontal lines of the toric diagram are effectively cut, since $h_{1, \ldots, 4} \rightarrow \infty$, while $v_{1, \ldots, 4}$ and $m_{1, \ldots, 4}$ remain finite. ${ }^{2}$ The remaining diagram takes the form of two vertical strips, ${ }^{3}$ thus implying that the gauge group associated with the horizontal expansion is

$$
G_{\mathrm{hor}}=U(2) \times U(2) .
$$

Indeed, $\rho-\hat{b}_{1}$ and $\hat{b}_{1}$ are related to the gauge couplings associated with each of the two $U(2)$ factors, while the parameters $\hat{c}_{1,2}$ can be interpreted as the (simple, positive) roots of each of two $\mathfrak{a}_{1}$ related to the two $U(2)$ factors. Furthermore, $\tau$ can be interpreted as an additional root, that extends each of these algebras to affine $\hat{\mathfrak{a}}_{1}$, while $E$ is a parameter associated with the compactification of the toric web on a torus.

(ii) vertical expansion in the basis $\left(\tau, \hat{c}_{1} ; \hat{b}_{1}, \hat{b}_{2} ; \rho, D\right)$

In the limit

$$
\tau-\hat{c}_{1} \rightarrow \infty, \quad \text { and } \quad \hat{c}_{1} \rightarrow \infty,
$$

all vertical lines of the toric diagram are cut, since $v_{1, \ldots, 4} \rightarrow \infty$, while $h_{1, \ldots, 4}$ and $m_{1, \ldots, 4}$ remain finite [and positive for certain values of $\left.\rho, \hat{b}_{1}, \hat{b}_{2}, D\right)$ ]. In this way, the diagram decomposes into two horizontal strips, which implies that the gauge group associated with the vertical expansion is, in fact,

\footnotetext{
${ }^{2}$ Notice, in particular, that there exists a region in the parameter space of $\left(\tau, \hat{c}_{1}, \hat{c}_{2}, E\right)$ in which $\left(v_{1}, v_{2}, v_{3}, v_{4}, m_{1}, m_{2}, m_{3}, m_{4}\right)$ are positive, which is important for their interpretation from the point of view of gauge theory as Coulomb branch parameters and hypermultiplet masses, respectively.

${ }^{3}$ While we call them strips, we point out that they are still defined on a cylinder; i.e., their ends are being identified (see also Fig. 3).
} 
TABLE I. Three different choices of maximal sets of independent Kähler parameters for the configuration $(N, M)=(2,2)$. In each case, the 12 lines $\left(h_{1, \ldots, 4}, v_{1, \ldots, 4}, m_{1, \ldots 4}\right)$ are parametrized by six independent variables. The last row shows the weak coupling limit, which is obtained by sending two of the parameters (related to the coupling constants of the respective gauge theories) to infinity.

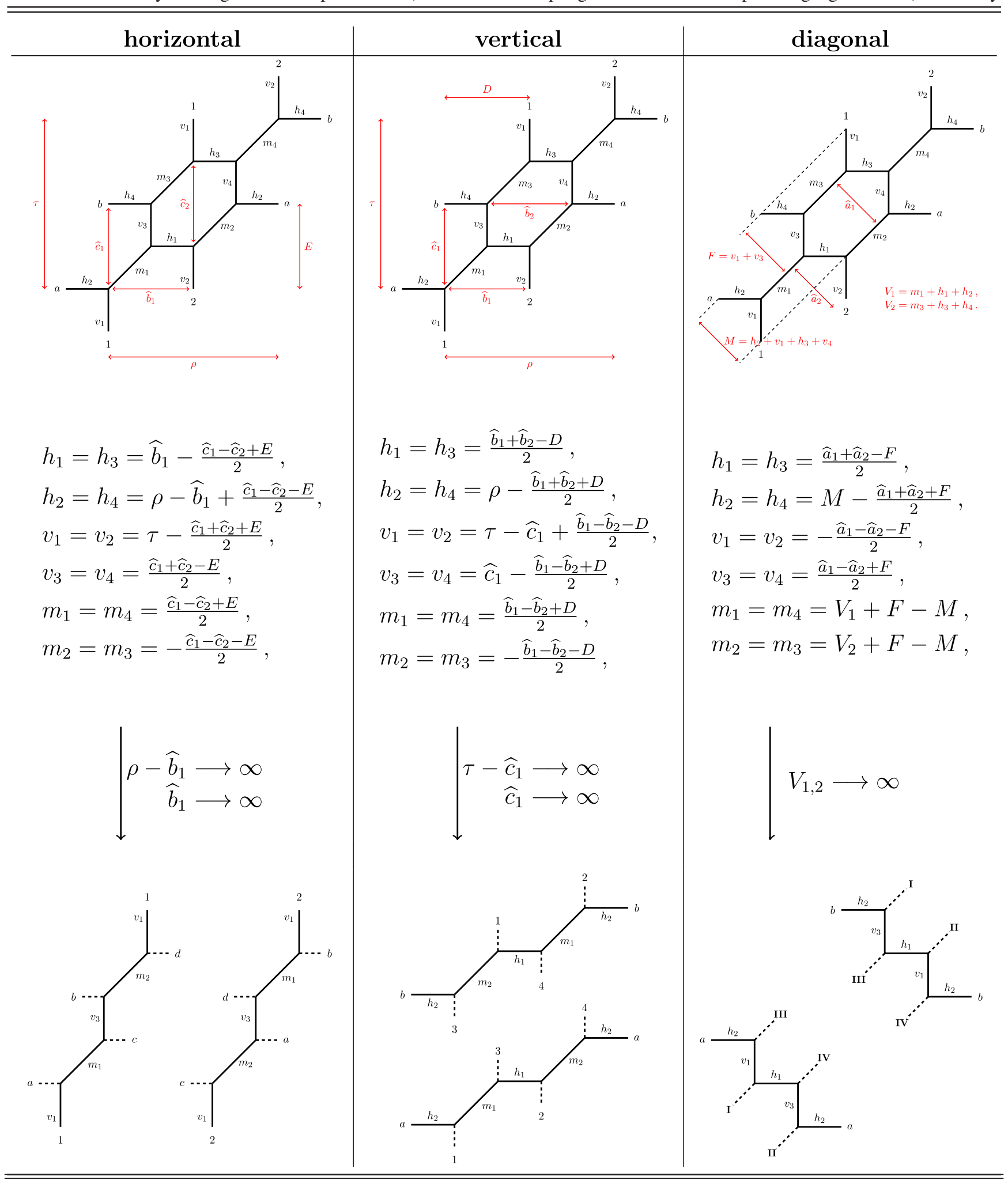




$$
G_{\mathrm{vert}}=U(2) \times U(2) .
$$

This group is in fact the electromagnetic S-dual (i.e., the Langlands dual) of the horizontal gauge group (3.2). Indeed, $\tau-\hat{c}_{1}$ and $\hat{c}_{1}$ can be related to the gauge couplings associated with each of the two $U(2)$ factors, and the parameters $\hat{b}_{1,2}$ can be interpreted as the (simple, positive) roots of each of two $\mathfrak{a}_{1}$ related to the two $U(2)$ factors. The parameter, $\tau$ can be interpreted as an additional root, that extends each of these algebras to affine $\hat{\mathfrak{a}}_{1}$, while $D$ is a parameter associated with the compactification of the toric web on a torus.

(iii) diagonal expansion in the basis $\left(V_{1}, V_{2} ; \hat{a}_{1}, \hat{a}_{2} ; F, L\right)$

In the limit $V_{1,2} \rightarrow \infty$, all diagonal lines [along direction $(1,1)]$ of the toric diagram are cut, since $m_{1, \ldots, 4} \rightarrow \infty$, while $h_{1, \ldots, 4}$ and $v_{1, \ldots, 4}$ remain finite (and positive for certain values). In this way, the diagram decomposes into two diagonal strips (which were called "staircase strips" in [36]), which implies that the gauge group associated with the vertical expansion is as well

$$
G_{\text {diag }}=U(2) \times U(2) .
$$

Indeed, $V_{1,2}$ can be related to the gauge couplings associated with each of the two $U(2)$ factors, and the parameters $\hat{a}_{1,2}$ can be interpreted as the (simple, positive) roots of each of two $\mathfrak{a}_{1}$ related to the two $U(2)$ factors. The parameter, $L$ can be interpreted as an additional root, that extends each of these algebras to affine $\hat{\mathfrak{a}}_{1}$, while $F$ is a parameter associated with the compactification of the toric web on a torus.

It is important to notice that in all three cases, the connection to a certain gauge theory relies on the fact that in the weak coupling limit the web diagram decomposes into a number of parallel strips (either horizontally, vertically or diagonally): physically, the latter can be interpreted as parallel NS5 branes with semi-infinite D5-branes ending on either side in equal numbers [64]. When the strips are glued together the world-volume theory on these D5-branes is the corresponding gauge theory, as explained above in Sec. II B 4.

In the current case, since the orientation of the strips can be changed through an $S L(2, \mathbb{Z})$ transformation, the diagrams in the last line of Table I are identical up to a relabeling of the parameters. This indicates that the gauge theories engineered from the three expansions in (2.21) have the same gauge group, i.e.,

$$
G_{\mathrm{hor}}=G_{\mathrm{vert}}=G_{\mathrm{diag}}=U(2) \times U(2) .
$$

This is a peculiarity of the configuration $(N, M)=(2,2)$ as in general the three gauge groups are different (albeit that their rank is the same as argued above), as we shall see from the next example $(N, M)=(3,2)$.

\section{B. Configuration $(N, M)=(3,2)$}

The next nontrivial configuration corresponds to $(N, M)=(3,2)$ with $k=\operatorname{gcd}(3,2)=1$. The corresponding web diagram contains 18 lines which are the Kähler parameters of various rational curves in the Calabi-Yau threefold $X_{3,2}$ :

$$
\begin{aligned}
\mathbf{h} & =\left(h_{1}, \ldots, h_{6}\right), \quad \mathbf{v}=\left(v_{1}, \ldots, v_{6}\right), \\
\mathbf{m} & =\left(m_{1}, \ldots, m_{6}\right) .
\end{aligned}
$$

As discussed before, these parameters are not linearly independent but they can be parametrized by choosing eight independent variables (for more details see also [36]). Three different such parametrizations are shown in Table II, leading to the following expansions:

(i) horizontal expansion in the basis $\left(\rho, \hat{b}_{1}, \hat{b}_{2} ; \hat{c}_{1}, \hat{c}_{2}, \hat{c}_{3}\right.$; $\tau, E)$

In the limit

$$
\rho-\hat{b}_{1}-\hat{b}_{2} \rightarrow \infty, \text { and } \hat{b}_{1} \rightarrow \infty, \quad \hat{b}_{2} \rightarrow \infty,
$$

we find $h_{1, \ldots, 6} \rightarrow \infty$, while $v_{1, \ldots, 6}$ and $m_{1, \ldots, 6}$ remain finite. Therefore, as indicated in Table II, in the limit (3.8) the toric web diagram decomposes into 3 vertical strips, implying that the horizontal expansion gives rise to a gauge theory with gauge group

$$
G_{\mathrm{hor}}=U(2) \times U(2) \times U(2),
$$

More specifically, the parameters $\left(\rho-\hat{b}_{1}-\hat{b}_{2}, \hat{b}_{1}, \hat{b}_{2}\right)$ are related to the gauge coupling constants, while $\hat{c}_{1}$, $\hat{c}_{2}$ and $\hat{c}_{3}$ can be interpreted as the (simple positive) roots of $\mathfrak{a}_{1}$ algebras associated with each of the $U(2)$ factors. Each of these algebras is further extended to affine $\hat{a}_{1}$ through the parameter $\tau$.

(ii) vertical expansion in the basis $\left(\tau, \hat{c}_{1} ; \hat{b}_{1}, \hat{b}_{2}\right.$, $\left.\hat{b}_{3}, \hat{b}_{4} ; \rho, D\right)$

In the limit

$$
\tau-\hat{c}_{1} \rightarrow \infty, \quad \hat{c}_{2} \rightarrow \infty,
$$

we find $v_{1, \ldots, 6} \rightarrow \infty$, while $h_{1, \ldots, 6}$ and $m_{1, \ldots, 6}$ remain finite. Therefore, the $(3,2)$ web diagram decomposes into two horizontal strips, indicating that the vertical expansion is associated with a gauge theory with gauge group

$$
G_{\text {vert }}=U(3) \times U(3) .
$$

In this manner, $\left(\tau-\hat{c}_{1}, \hat{c}_{1}\right)$ are related to the coupling constants and $\left(\hat{b}_{1}, \hat{b_{2}}\right)$ and $\left(\hat{b}_{3}, \hat{b}_{4}\right)$ correspond 
TABLE II. Three different parametrizations of the web diagram $(N, M)=(3,2)$. The last line shows the decomposition of the diagram in the weak coupling limit in the horizontal, vertical, and diagonal description, respectively.
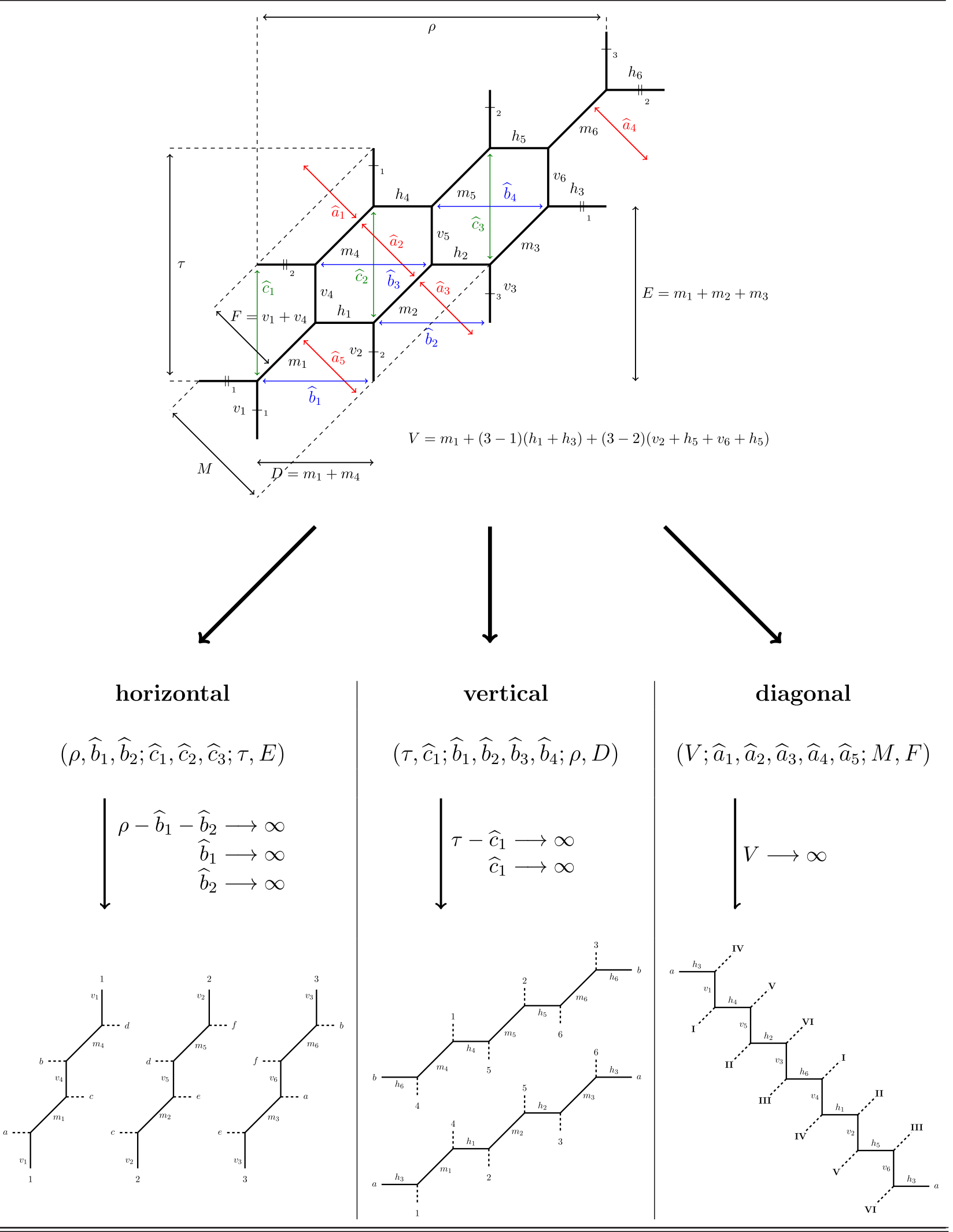

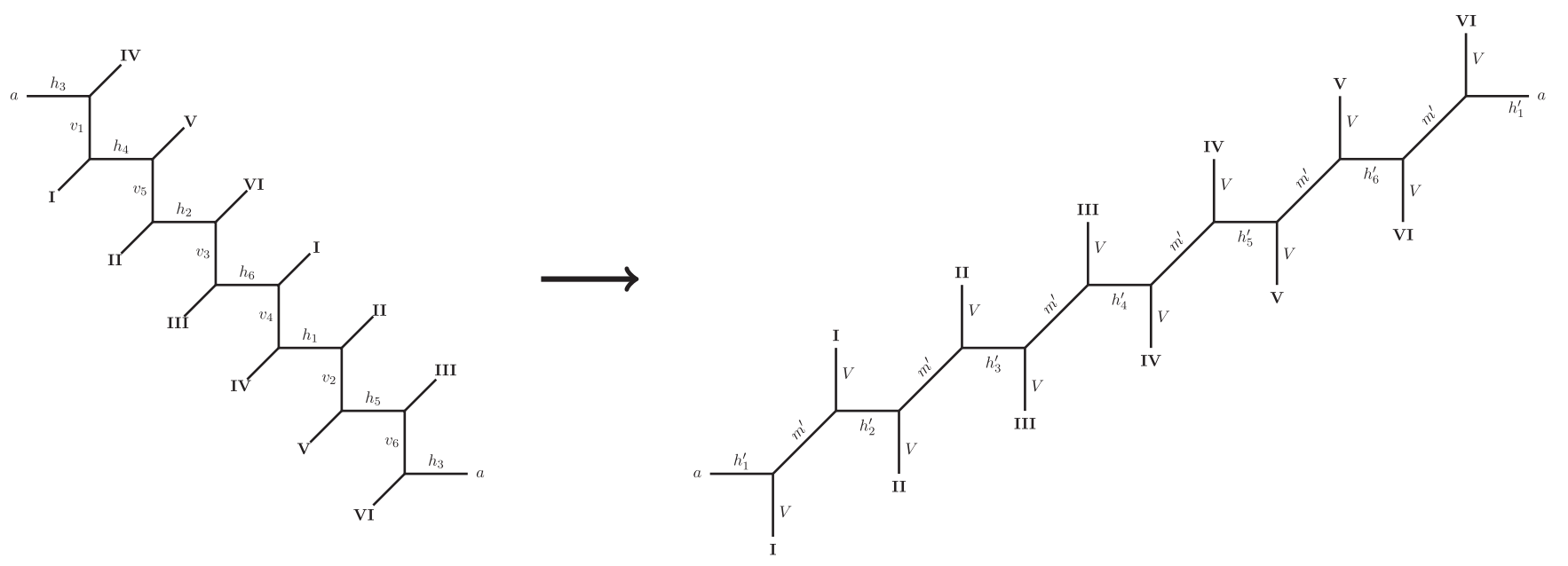

FIG. 6. Flop and symmetry transformations relating the diagonal presentation (rightmost column in Table II) to the toric web of $X_{6,1}$.

to the (simple positive) roots of two copies of $\mathfrak{a}_{2}$, associated with the to the $U(3)$ factors in (3.11). These algebras are extended to affine $\hat{\mathfrak{a}}_{2}$ by the parameter $\rho$.

(iii) diagonal expansion in the basis ( $V$; $\left.\hat{a}_{1}, \hat{a}_{2}, \hat{a}_{3}, \hat{a}_{4}, \hat{a}_{5} ; M, F\right)$

In the limit $V \rightarrow \infty$ we find $m_{1, \ldots .6} \rightarrow \infty$, while $h_{1, \ldots, 6}$ and $v_{1, \ldots, 6}$ remain finite, such that the web diagram decomposes into a single diagonal strip. This indicates that the diagonal expansion is associated with a gauge theory with gauge group

$$
G_{\text {diag }}=U(6) .
$$

Here $V$ is related to the coupling constant, while $\left(\hat{a}_{1}, \ldots, \hat{a}_{5}\right)$ play the role of the (simple positive) roots of $\mathfrak{a}_{5}$ associated with $G_{\text {diag }}$.

The fact that such a gauge theory exists also outside of the weak coupling limit $V \rightarrow \infty$ can be inferred from the duality of the web diagram shown in Table II with the toric web of $X_{6,1}$ through a series of flop and symmetry transformations. The latter was first discussed in [11] and is schematically shown in Fig. 6. In this duality transformation, the diagonal lines in the left part of the Fig. 6 do not undergo flop transitions, such that the Kähler parameters $h_{1,2,3,4,5,6}^{\prime}$ and $m^{\prime}$ of $X_{6,1}$ are independent of $V$. Therefore, the vertical expansion of the partition function $\mathcal{Z}_{6,1}$ is a power series in $Q_{V}=e^{-V}$, which can be interpreted as the instanton partition function $Z_{\mathrm{vert}}^{(6,1)}$ of a gauge theory with gauge group $U(6)$. Moreover, through the duality map implied by Fig. $6, Z_{\text {vert }}^{(6,1)}$ can also be related to $Z_{\text {diag }}^{(3,2)}$

$Z_{\mathrm{vert}}^{(6,1)}\left(V, h_{1, \ldots, 6}^{\prime}, m\right)=Z_{\mathrm{diag}}^{(3,2)}\left(V, \hat{a}_{1, \ldots, 5}, M, F\right)$, which was proven explicitly in [36]. This shows that $Z_{\text {diag }}^{(3,2)}$ (as a power series expansion in $Q_{V}$ ) can be read as the instanton partition function of a gauge theory with gauge group $U(6)$ also outside of the region $V \rightarrow \infty$.

In the case of $(N, M)=(3,2)$, the gauge groups $G_{\text {hor }}$, $G_{\text {vert }}$ and $G_{\text {diag }}$ are different ${ }^{4}$ however, as discussed in the previous section, their rank is identical.

Furthermore, we stress that in all three cases the specific form of the parametrization is not unique: Different choices of parameters leading to the same decomposition of the toric web diagram as in Table II are possible. Indeed, in [36], we have chosen a slightly different parametrization suitable for the explicit computations of the general building block of the partition function.

\section{General web configuration}

The discussion of the previous examples $(2,2)$ and $(3,2)$ can be generalized to a web diagram with generic $(N, M)$. Indeed, in the following, we make a proposal for three different parametrizations of the Kähler moduli space of $X_{N, M}$, facilitating the three expansions of $\mathcal{Z}_{N, M}$ that were schematically written in (2.21). In the following, we present sets-in general not unique- of $N M+2$ independent parameters (which we shall refer to as a basis in the following) suitable for the description of the horizontal, vertical and diagonal theory. ${ }^{5}$

The geometric interpretation of (some of) the parameters in the bases is shown in Fig. 7. The orange box in Fig. 7

\footnotetext{
${ }^{4}$ We remark, however, that at a generic point in moduli space, the gauge groups are broken down to $U(1),{ }^{6}$ respectively.

${ }^{5}$ This choice of bases is motivated by studying numerous examples with small values of $N$ or $M$ such as those in Secs. III A and III B. A proof of the linear independence for generic $(N, M)$ is currently still missing.
} 
highlights a generic hexagon in the $(N, M)$ web diagram, which can be labeled by two integers

$$
\begin{aligned}
r & \in\{0,1 \ldots, M-1\} \bmod M, \\
\text { and } \quad s & \in\{1, \ldots, N\} \bmod N .
\end{aligned}
$$

With the parameters shown in Fig. 7, we propose the following three (inequivalent) bases

(i) horizontal basis

We propose as a basis suitable for the description of the horizontal expansion $Z_{\mathrm{hor}}^{(N, M)}$ in (2.21) the following

$$
\mathfrak{B}_{\text {hor }}=\left\{\hat{b}_{M-1, s=1, \ldots, N-1}, \rho,\{\hat{c}\}_{u=1, \ldots, N}, \tau, E\right\},
$$

with

$$
\begin{aligned}
\{\hat{c}\}_{u}= & \left\{\hat{c}_{r, u} \mid r \in\{0,1, \ldots, M-2\}\right\}, \\
& \forall u=1, \ldots, N .
\end{aligned}
$$

This basis indeed suggests that $Z_{\text {hor }}^{(N, M)}$ is the instanton partition function of gauge theory with gauge group $G_{\text {hor }}=[U(M)]^{N}$ : indeed $\{c\}_{u=1, \ldots, N}$ furnish $N$ sets of (simple positive) roots for the $N$ factors of $U(M)$, while the $N$ decoupling parameters,

$$
\left\{\hat{b}_{M-1,1}, \hat{b}_{M-1,2}, \ldots, \hat{b}_{M-1, N-1}, \rho-\sum_{i=1}^{N-1} \hat{b}_{M-1 i}\right\}
$$

are related with the gauge coupling constants as mentioned in Eq. (2.15) (one associated with every factor of $U(M)$ in $G_{\mathrm{hor}}$ ), in the sense ${ }^{6}$ that in the limit

$$
\begin{aligned}
\rho-\sum_{i=1}^{N-1} \hat{b}_{M-1, i} & \rightarrow \infty, \quad \text { and } \\
\hat{b}_{M-1, i} & \rightarrow \infty \quad \forall i=1, \ldots, N-1,
\end{aligned}
$$

we have $h_{1, \ldots, N M} \rightarrow \infty$, while $\left\{v_{1, \ldots, N M}, m_{1, \ldots, N M}\right\}$ in the diagram in Fig. 7 remain finite. Graphically, the diagram therefore composes into $N$ vertical strips of length $M$, each of which associated with the theory corresponding to a single $U(M)$. The expansion of the partition function $Z_{\mathrm{hor}}^{(N, M)}$ [schematically written in (2.21)] can therefore be more concisely be written as an instanton expansion in (3.17).

\footnotetext{
${ }^{6}$ Notice, depending on the explicit realization of the gauge theory, the coupling constants can be shifted by some of the remaining parameters as long as they lead to the same weak coupling theory. We will be more specific on this point later on, when we discuss explicit examples.
}

Finally, the parameter $\tau$ extends each of the algebras $\mathfrak{a}_{M-1}$ [whose roots are given in (3.16)] to affine $\hat{\mathfrak{a}}_{M-1}$.

(ii) vertical basis

A basis suitable for describing the vertical expansion $Z_{\mathrm{vert}}^{(N, M)}$ in (2.21) can be found through a judicious exchange of vertical and horizontal parameters of the horizontal basis. Indeed, we propose the vertical basis to be

$$
\mathfrak{B}_{\mathrm{vert}}=\left\{\hat{c}_{r=0, \ldots, M-2, N}, \tau,\{\hat{b}\}_{u=0, \ldots, M-1}, \rho, D\right\},
$$

with

$$
\begin{gathered}
\{\hat{b}\}_{u}=\left\{\hat{b}_{u, s} \mid s \in\{1, \ldots, N-1\}\right\}, \\
\forall u=0, \ldots, M-1,
\end{gathered}
$$

which suggests that $Z_{\mathrm{vert}}^{(N, M)}$ can be interpreted as the instanton partition function of a gauge theory with gauge group $G_{\text {vert }}=[U(N)]^{M}$. Specifically $\{b\}_{u=0, \ldots, M-1}$ furnish $M$ sets of (simple positive) roots for the $M$ different factors of $U(N)$. Moreover, the $M$ parameters

$$
\left\{\hat{c}_{0, N}, \ldots, \hat{c}_{M-2, N}, \tau-\sum_{i=0}^{M-2} \hat{c}_{i, N}\right\}
$$

are the decoupling parameters [Eq. (2.15)] which are associated with the gauge coupling constants [one associated with each of the $M$ factors $U(N)]$ in the sense that in the limit

$$
\begin{aligned}
\tau & -\sum_{i=0}^{M-2} \hat{c}_{i, N} \rightarrow \infty, \\
\text { and } \quad \hat{c}_{i, N} & \rightarrow \infty \quad \forall i=0, \ldots, M-2,
\end{aligned}
$$

we have $v_{1, \ldots, N M}$ while $\left\{h_{1, \ldots, N M}, m_{1, \ldots, N M}\right\}$ remain finite. Thus, the diagram in Fig. 7 therefore decomposes into $M$ horizontal strips of length $N$ each of which begin associated with the theory corresponding to a single $U(N)$. The series expansion $Z_{\mathrm{vert}}^{(N, M)}$ [which is schematically given in (2.21)] can therefore be more concisely be written as an instanton expansion in (3.21).

Finally, the parameter $\rho$ extends each of algebras $\mathfrak{a}_{N-1}$ [whose roots are given in (3.20)] to affine $\hat{\mathfrak{a}}_{N-1}$.

(iii) diagonal basis

The diagonal expansion is somewhat more involved to describe. Indeed, we propose the following $N M+2$ parameters as a basis [with $k=\operatorname{gcd}(N, M)$ ] for the diagonal expansion 


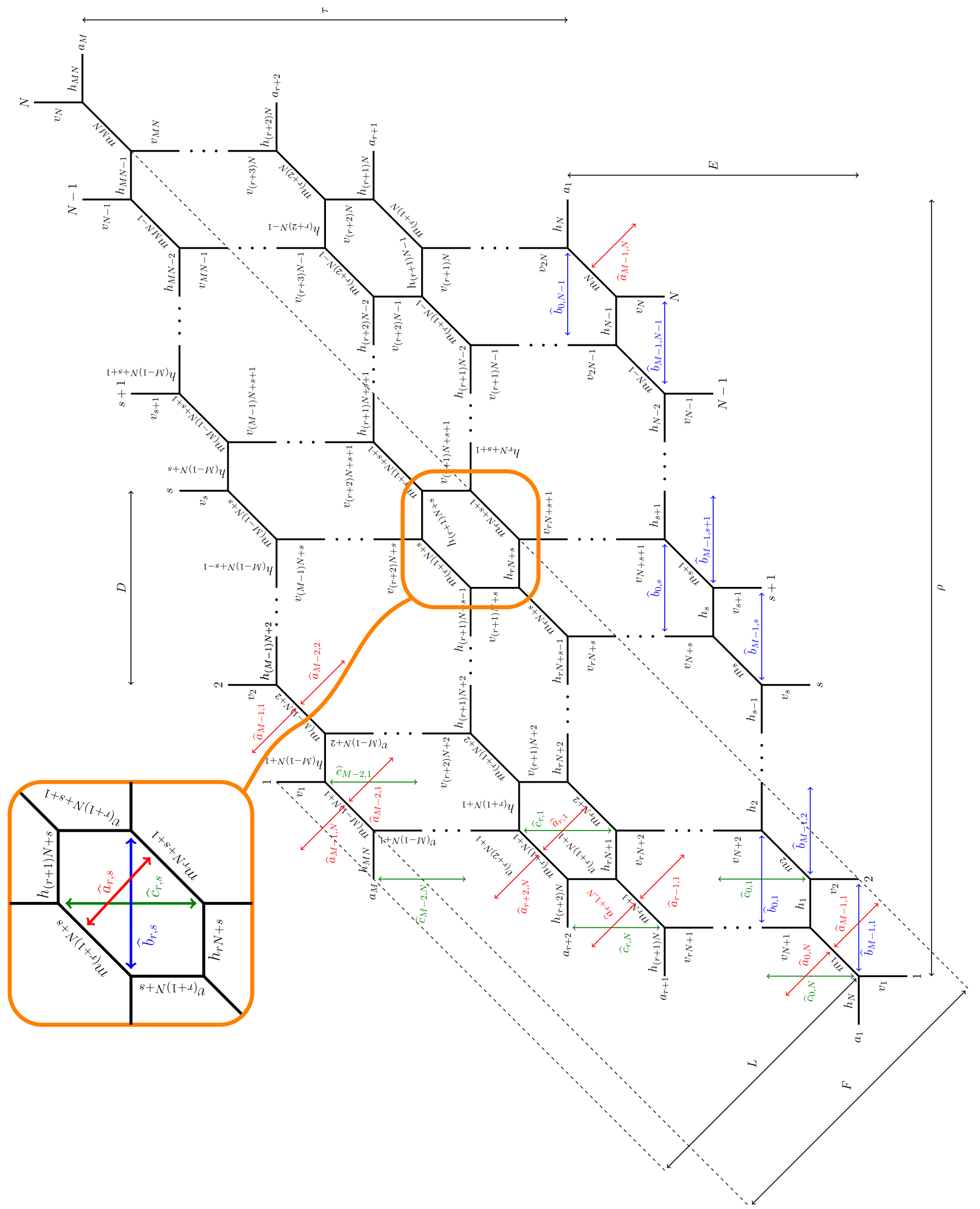

FIG. 7. Three different maximally independent sets of Kähler parameters for a generic toric web $(N, M)$. For concreteness, we assume $N \geq M$. Furthermore, for the sets $\hat{a}, \hat{b}$ and $\hat{c}$ (which will constitute the roots in the three different gauge theory descriptions), we have only shown the first few explicitly in the diagram, along with an assignment for a generic hexagon in the web. The latter is labeled by two integers $(r, s)$ whose range is specified in Eq. (3.14). 
$\mathfrak{B}_{\text {diag }}=\left\{V_{1}, \ldots, V_{k},\{\hat{a}\}_{u=0, \ldots, k-1}, L, F\right\}$,

with

$$
\{\hat{a}\}_{u}=\left\{\hat{a}_{M-1-a-u, N+a} \mid a \in\left\{0, \ldots, \frac{M N}{k}-2\right\}\right\},
$$

which suggest that $Z_{\mathrm{diag}}^{(N, M)}$ in (2.21) is the instanton partition function of a gauge theory with gauge group $G_{\text {diag }}=[U(N M / k)]^{k}$. In (3.23), the parameters $V_{1, \ldots, k}$ are difficult to directly identify in the web diagram in Fig. 7. They can, however, be written as a linear combination of $(\mathbf{h}, \mathbf{v}, \mathbf{m})$. To this end, we introduce a similar notation as in [11]: for any diagonal line of area $m_{a}$ (with $a=1, \ldots, N M$ ) stretched between two vertices $A$ and $B$

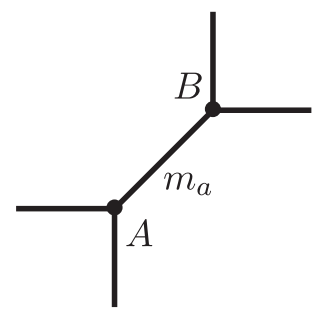

we define $\mathcal{P}_{L}\left(m_{a}\right)$ as the path starting at $A$ and following $N$ distinct horizontal and $N-1$ distinct vertical lines (going to the left), as well as $\mathcal{P}_{R}\left(m_{a}\right)$ the path starting at $B$ and following $N$ distinct horizontal and $N-1$ distinct vertical lines (going to the right). Furthermore, we denote $\left(\mathfrak{p}_{L}\left(m_{a}\right)\right)_{i=1, \ldots, 2 N-1}$ and $\left(\mathfrak{p}_{R}\left(m_{a}\right)\right)_{i=1, \ldots, 2 N-1}$ as the components of $\mathcal{P}_{L}\left(m_{a}\right)$ and $\mathcal{P}_{R}\left(m_{a}\right)$, respectively. ${ }^{7}$ With this notation, we define ${ }^{8}$ the decoupling parameters [Eq. (2.15)] $(a=0, \ldots, k)$

$$
\begin{aligned}
V_{a+1}= & m_{1+a N}+\left(\frac{N}{k}-1\right)\left(\left(\mathfrak{p}_{L}\left(m_{1+a N}\right)\right)_{1}+\left(\mathfrak{p}_{R}\left(m_{1+a N}\right)\right)_{1}\right) \\
& +\sum_{i=1}^{\frac{N}{k}-2}\left(\frac{N}{k}-1-i\right)\left[\left(\mathfrak{p}_{L}\left(m_{1+a N}\right)\right)_{2 i}+\left(\mathfrak{p}_{R}\left(m_{1+a N}\right)\right)_{2 i}\right] \\
& +\sum_{i=1}^{\frac{N}{k}-2}\left(\frac{N}{k}-1-i\right)\left[\left(\mathfrak{p}_{L}\left(m_{1+a N}\right)\right)_{2 i+1}\right. \\
& \left.+\left(\mathfrak{p}_{R}\left(m_{1+a N}\right)\right)_{2 i+1}\right]
\end{aligned}
$$

\footnotetext{
${ }^{7}$ We refer the reader to Sec. V of [11], pointing out, however, that in the latter work $N<M$ had been assumed such that the roles of the horizontal and vertical lines have been exchanged.

${ }^{8}$ While the definition (3.26) is very abstract, it is inspired by the definition of the gauge coupling constants of the vertical expansion associated with the dual Calabi-Yau $X_{\frac{N M}{k}, k}$ as explained in [11].
}

Indeed, for $V_{1, \ldots, k} \rightarrow \infty$ we have $m_{1, \ldots, N M} \rightarrow \infty$, while $\left(h_{1, \ldots, M N}, v_{1, \ldots, M N}\right)$ remain finite. In this way, the $(N, M)$ web diagram decomposes into $k$ diagonal strips of length $\frac{N M}{k}$, which can be interpreted as the weak coupling limit of a quiver gauge theory whose gauge group is $G_{\text {diag }}=$ $\left[U\left(\frac{N M}{k}\right)\right]^{k}{ }^{9}$. The existence of this theory outside of the weak coupling limit can be argued by the fact that $X_{N, M}$ is dual to $X_{N M / k, k}$ through a combination of flop and symmetry transformations proposed in [11]. Throughout this series of transformations, the diagonal lines (labeled by $\left.m_{1, \ldots N M}\right)$ do not undergo flop transitions, such that the $V_{1, \ldots, k}$ are related to the coupling constants of the $\left[U\left(\frac{N M}{k}\right)\right]^{k}$ quiver gauge theory furnished by the vertical expansion of $\mathcal{Z}_{N M / k, k}$. Moreover, due to the fact that the partition function is expected to be invariant under the duality proposed in [11] (this was explicitly proven for $k=1$ in [36]), we propose that the expansion of $\mathcal{Z}_{N, M}$ in powers of $Q_{V_{a}}=e^{-V_{a}}$ (for $a=1, \ldots, k$ ) can also be interpreted as the instanton partition function of a quiver gauge theory with gauge group $\left[U\left(\frac{N M}{k}\right)\right]^{k}$. From this perspective, the $\{\hat{a}\}_{u=0, \ldots, k-1}$ in (3.24) furnish $k$ sets of (simple positive) roots, each associated with a factor $U\left(\frac{N M}{k}\right) \subset G_{\text {diag }}$.

Finally, the parameter $L$ extends each of algebras $\mathfrak{a}_{N M / k-1}$ [whose roots are given in (3.24)] to affine $\hat{\mathfrak{a}}_{N M / k-1}$.

To summarize, based on the proposed bases $\mathfrak{B}_{\text {hor }}, \mathfrak{B}_{\text {vert }}$ and $\mathfrak{B}_{\text {diag }}$ (as well as the examples discussed above) we conjecture that for given $(N, M)$ we can engineer three different gauge theories

(i) horizontal gauge theory with gauge group $G_{\mathrm{hor}}=$ $[U(M)]^{N}$

(ii) vertical gauge theory with gauge group $G_{\text {vert }}=$ $[U(N)]^{M}$

(iii) diagonal gauge theory with gauge group $G_{\text {diag }}=$ $[U(N M / k)]^{k}$ with $k=\operatorname{gcd}(N, M)$

whose gauge groups have the same rank. Moreover, since the partition functions of these three theories are identical (indeed, by construction they are simply different expansions of $\mathcal{Z}_{N, M}$, namely $\mathcal{Z}_{\text {hor }}^{(N, M)}, \mathcal{Z}_{\text {vert }}^{(N, M)}$, and $\mathcal{Z}_{\text {diag }}^{(N, M)}$, respectively) they are mutually dual to each other leading to the triality

$$
\begin{aligned}
G_{\mathrm{hor}} & =[U(M)]^{N} \leftrightarrow G_{\mathrm{vert}}=[U(N)]^{M} \leftrightarrow G_{\mathrm{diag}} \\
& =[U(M N / k)]^{k} .
\end{aligned}
$$

Notice that this duality is not limited to the weak coupling limit: by decomposing the web diagram in parallel strips (whose explicit form was recently computed in [36] in full generality), the triality can be extended to the full nonperturbative partition function.

\footnotetext{
${ }^{9}$ Each strip can be associated with an individual $U\left(\frac{N M}{k}\right) \subset G_{\text {diag }}$.
} 


\section{TRIALITY AND W-ALGEBRAS}

In this section, we briefly discuss the implication of the triality of gauge theories, that we discussed in the previous sections, for the $\mathrm{W}$-algebras. Recall that the six-dimensional $(2,0)$ theory can be compactified on a Riemann surface $\Sigma$ with punctures to obtain the four-dimensional class $\mathcal{S}$ theories [65]. Since the $A_{N-1}$ type six-dimensional $(2,0)$ theory can be realized on the world volume of the $N$ coincident M5-branes, we can take the world volume to be

$$
\mathbb{R}_{\epsilon_{1}, \epsilon_{2}}^{4} \times \Sigma
$$

to obtain an $\Omega$ deformed four-dimensional gauge theory whose details such as the gauge group and the matter content depend on the details of $\Sigma$ (its punctures and its pair of pants decomposition). In [37], it was conjectured (the AGT conjecture) that the compactification of the $A_{1}(2,0)$ theory on the Riemann surface $\Sigma$ gives rise to Liouville theory such that the instanton partition function of the four-dimensional theory can be obtained from the conformal blocks of the two-dimensional Liouville theory. The conjecture was extended to the case of $A_{N-1}(2,0)$ six-dimensional theory in which case the two-dimensional theory on the Riemann surface is the $A_{N-1}$ Toda theory [38]. It was shown in [66] that the AGT conjecture and its extension to the $A_{N-1}$ case can be understood using B-model topological strings.

The two-dimensional theory on the Riemann surface has the Virasoro symmetry for the $N=2$ case which generalizes to $W_{N}$ for the general $A_{N-1}$. In lifting the fourdimensional instanton counting to five dimensions, with $\mathcal{N}=1$ theory on $\mathbb{R}^{4} \times S^{1}$, a q-deformation is introduced which corresponds to considering the K-theory of instanton moduli spaces rather than the cohomology. It was shown in [39-41] that the q-deformed instanton counting or the K-theoretic instanton partition functions $[30,67]$ satisfy the q-deformed $W_{N}$ constraints. More recently it was shown in [68] that for a quiver gauge theory with quiver $\Gamma$ the partition function can be written as a correlation function in the free field representation of the $W(\Gamma)$ algebra. Lifting it further to six dimensions gives an elliptic deformation of the instanton counting which can be related to the elliptic deformation of the Virasoro and the $W_{N}$ algebras [69-73].

There was a parallel development on the mathematics side in the study of geometry of instanton moduli spaces which was greatly helped by the AGT conjecture. Maulik-Okounkov [74] and Schiffmann-Vesserot [75] proved that for the instanton moduli space of type $G, M_{G}$, one can construct an action of the W-algebra of type $G$ on the cohomology of $M_{G}$ such that the unit cohomology class is related to the Gaiotto state and its pairing with itself gives the Nekrasov instanton partition function. It was shown in [76,77] that the construction of Maulik-Okounkov and Schiffmann-Vesserot can be realized for the K-theory of the instanton moduli spaces giving the analog of the AGT conjecture for the five-dimensional $\mathcal{N}=1$ theories.

In [78], $\mathcal{N}=2$ the Seiberg-Witten geometry of the fourdimensional gauge theories with gauge group given by a

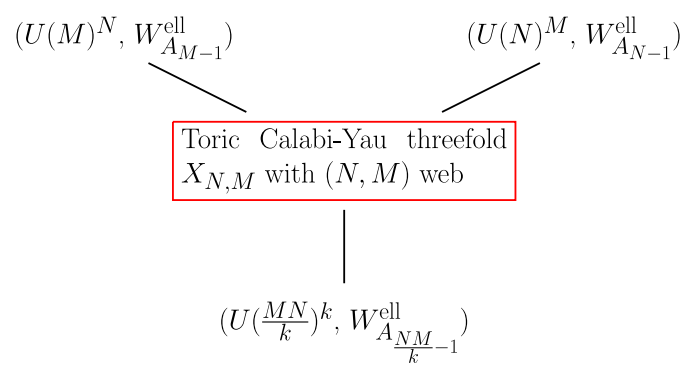

FIG. 8. The gauge groups and W-algebras associated to the gauge theories coming from the $(N, M)$ web. There are potentially more branches of this tree depending on the product $N M$ and the $\operatorname{gcd}(N, M)$ as discussed in previous sections.

quiver were studied. As was discussed in [79] some of these theories, depending on the quiver and the gauge group factors associated with the nodes of the quiver, can be realized geometrically in terms of Calabi-Yau threefolds and are dual to other gauge theories via the fiber-base duality. It was argued in [80-82] that because of the fiber base duality two different $\mathrm{W}$-algebras can be associated with the gauge theory. If we denote the gauge group of the two dual theories by $G_{1}$ and $G_{2}$, respectively, then both theories realize $W_{G_{1}}$ and $W_{G_{2}}$. For theories coming from six-dimensional theories on $T^{2}$, the $\mathrm{W}$-algebra is deformed to the elliptic $\mathrm{W}$-algebras [73]. The triality we discussed in the previous sections extends the class of (elliptic) W-algebra associated with a theory by identifying new dual theories. From the results of the previous sections it follows that if we consider the $(N, M)$ web then associated to it are several dual gauge theories, as shown in Fig. 8, and hence several elliptic Walgebras. Since these theories have the same partition function $\mathcal{Z}_{N, M}$, which is the topological string partition function of $X_{N, M}$, with gauge theory parameters associated with Kähler parameters of $X_{N, M}$ in different ways therefore the identification of generators of different elliptic $\mathrm{W}$-algebras is in terms of distinct parameters [43].

\section{CONCLUSIONS}

In this paper, we have analyzed dualities of a class of little string orbifold theories with eight supercharges as well as the supersymmetric gauge theories given by their lowenergy limit below the string tension scale. Among other approaches, the (nonperturbative) gauge theory partition functions are captured by the refined topological string partition function $\mathcal{Z}_{N, M}\left(\mathbf{h}, \mathbf{v}, \mathbf{m}, \epsilon_{1,2}\right)$ of a class of toric Calabi-Yau threefolds $X_{N, M}$, whose generic web diagram is shown in Fig. 1. The latter consists of three sets of parallel lines whose associated Kähler parameters are denoted collectively by $\{\mathbf{h}\},\{\mathbf{v}\}$, and $\{\mathbf{m}\}$, respectively. ${ }^{10} \mathrm{We}$

\footnotetext{
${ }^{10}$ Of these $3 N M$ parameters, however, only $N M+2$ are independent of each other.
} 
have argued that for a given such toric web there exist three (generically distinct) dual $(p, q)$ 5-brane web descriptions in type II string theory in which each of these three sets of parallel lines is identified with the D5-branes, respectively. In each of these three brane configurations, the worldvolume theory on the latter can be identified with a quiver gauge theory. Therefore, we find a triality of gauge theories associated with a given toric Calabi-Yau manifold $X_{N, M}$, whose gauge groups are

$$
\begin{aligned}
G_{\mathrm{hor}} & =[U(M)]^{N}, \quad \text { and } G_{\mathrm{vert}}=[U(N)]^{M}, \\
\text { and } \quad G_{\mathrm{diag}} & =[U(M N / k)]^{k} .
\end{aligned}
$$

The duality between the horizontal and vertical theory has been discussed previously in the literature $[22,27,29]$ and is a consequence of an $S L(2, \mathbb{Z})$ symmetry of type IIB string theory. However, to give evidence for the full triality (including, in particular, the diagonal theory) we have argued $^{11}$ for the existence of three different regions in the Kähler cone of $X_{N, M}$ in which the toric web diagram decomposes into a number of horizontal, vertical or diagonal strips, respectively. From the point of view of the $(p, q)$ fivebrane theories, a strip correspond to the NS5-brane with equal number of D5-branes ending on it from either side. Since the distance between the NS5-branes correspond to the inverse gauge coupling the configuration of disconnected strips is therefore identified with the weak coupling limit of the associated world-volume theory. More precisely, for each of these three descriptions, we have identified the Kähler parameters of the toric web of $X_{N, M}$ (see (3.17), (3.21), and (3.26), respectively) that govern the distances of the NS5-branes in the brane web picture and which are related to the inverse coupling constants of the respective gauge theories. In turn, expanding $\mathcal{Z}_{N, M}\left(\mathbf{h}, \mathbf{v}, \mathbf{m}, \epsilon_{1,2}\right)$ as a power series in (the exponential of) these parameters, respectively, can be interpreted as the Nekrasov instanton partition function of the three gauge theories (5.1), as explained in (2.21). From the point of view of the (refined) topological vertex [which is used to compute $\mathcal{Z}_{N, M}(\mathbf{h}$, $\left.\left.\mathbf{v}, \mathbf{m}, \epsilon_{1,2}\right)\right]$ these three different expansions correspond to choosing a preferred direction in the web diagram (which can either be horizontally, vertically or diagonally). The diagonal expansion has recently been discussed in [36], where moreover a generic building block has been computed that allows to determine these three expansions of $\mathcal{Z}_{N, M}\left(\mathbf{h}, \mathbf{v}, \mathbf{m}, \epsilon_{1,2}\right)$ explicitly for generic $(N, M)$.

For the sake of clarity, we provide an overview over the three different gauge theories (along with an overview of which Kähler parameters of $X_{N, M}$ govern the coupling

\footnotetext{
${ }^{11}$ We have supplemented our reasoning by a number of examples in which the existence of these regions was shown explicitly.
}

constants and constitute the Coulomb branches and hypermultiplet masses, respectively) in the following table.

\begin{tabular}{lccccc}
\hline Theory & $\begin{array}{c}\text { Partition } \\
\text { function }\end{array}$ & $\begin{array}{c}\text { Gauge } \\
\text { group }\end{array}$ & $\begin{array}{c}\text { Decoupling } \\
\text { parameters }\end{array}$ & $\begin{array}{c}\text { Coulomb } \\
\text { branch }\end{array}$ & $\begin{array}{c}\text { Hypermultiplet } \\
\text { masses }\end{array}$ \\
\hline Horizontal & $Z_{\text {hor }}^{(N, M)}$ & {$[U(M)]^{N}$} & Equation (3.17) & $\{\mathbf{v}\}$ & $\{\mathbf{m}\}$ \\
Vertical & $Z_{\text {vert }}^{(N, M)}$ & {$[U(N)]^{M}$} & Equation (3.21) & $\{\mathbf{h}\}$ & $\{\mathbf{m}\}$ \\
Diagonal & $Z_{\text {diag }}^{N, M)}$ & {$\left[U\left(\frac{N M}{k}\right)\right]^{k}$} & Equation (3.26) & $\{\mathbf{h}, \mathbf{v}\}$ & $\{\mathbf{h}, \mathbf{v}\}$ \\
\hline
\end{tabular}

From the point of view of the gauge theories, however, the above table in general does not exhaust all possible dual theories. As will be discussed in [43] a larger set of dual theories can be found by studying the extended moduli space associated with $X_{N, M}$.

It would be interesting to extend the web of dualities we have found to other classes of little string theories (or supersymmetric gauge theories). The theories we have studied in this paper can be geometrically realized as resolutions of a $\mathbb{Z}_{N} \times \mathbb{Z}_{M}$ orbifold of $X_{1,1}$, which is a toric Calabi-Yau manifold that resembles the conifold in a certain decompactification limit. The dualities we have studied here, are based on the fact that different superconformal gauge theories can be associated with different resolutions of these A-type singularities, which, however, all lie in a common (extended) moduli space. They can therefore be related through various symmetry transformations or geometric transitions. It is an interesting question, whether similar relations can be obtained for different types of orbifolds, where $\mathbb{Z}_{N} \times \mathbb{Z}_{M}$ is replaced by $G_{1} \times G_{2}$, with $G_{i}$ some other discrete subgroup of $S U(2)$.

It would also be interesting to compactify the theories studied in this work to four-dimensional $\mathcal{N}=2$ supersymmetric gauge theories. As long as the compactification keeps the Coulomb branch compact, these theories would also exhibit an extensive web of dualities: the duality between the theories with $[U(M)]^{N}$ and $[U(N)]^{M}$ gauge group is known to correspond to exchange of quiver group and gauge group. The extended duality that encompasses $[U(M N / k)]^{k}$ is to our knowledge new.

\section{ACKNOWLEDGMENTS}

We are grateful to Dongsu Bak, Kang-Sin Choi, Taro Kimura, Jeong-Hyuck Park, Washington Taylor, and the participants of the Focus Program "Liouville, Integrability and Branes (13)" of the Asia-Pacific Center for Theoretical Physics for useful discussions. A.I. would like to acknowledge the "2017 Simons Summer Workshop on Mathematics and Physics" for hospitality during this work. A. I. was supported in part by the Higher Education Commission Grant No. HEC-20-2518.

\section{APPENDIX: SPECIAL CASES: SELF-TRIALITY AND SCFT LIMIT}

In Secs. III and IV, we have discussed different ways of associating gauge theories to a generic web diagram 
characterized by the parameters $(N, M)$. In general, the three gauge theories with gauge groups given in (3.27),

$$
\begin{aligned}
G_{\mathrm{hor}} & =[U(M)]^{N}, \quad \text { and } \quad G_{\text {vert }}=[U(N)]^{M}, \\
\text { and } \quad G_{\text {diag }} & =\left[U\left(\frac{M N}{k}\right)\right]^{k},
\end{aligned}
$$

are distinct, as can be seen by the fact the gauge groups are typically different. However, for particular web configurations the gauge groups of two or even all three of these gauge theories may become identical and the latter can be related to one another through a mere exchange of the parameters involved. In this sense some of these theories may be self-dual. In the following, we shall list examples of configurations in which this indeed happens.

\section{Web diagrams $(N, N)$}

A class of examples in which horizontal, vertical, and diagonal expansion yield gauge theories with the same gauge group (albeit with a different choice of parameters) are the configurations with $M=N$, giving rise to gauge theories with gauge group $[U(N)]^{N}$. This is obvious for the vertical and horizontal points of view. However it is less obvious for the diagonal point of view. Below, we show that the diagonal description again yields $[U(N)]^{N}$ quiver gauge theory, but the parameters are different from either the vertical or horizontal description.

Starting from the $(N, N)$ web diagram, the idea is that there always exists a way to cut $N$ diagonal lines simultaneously, as shown in the figure below

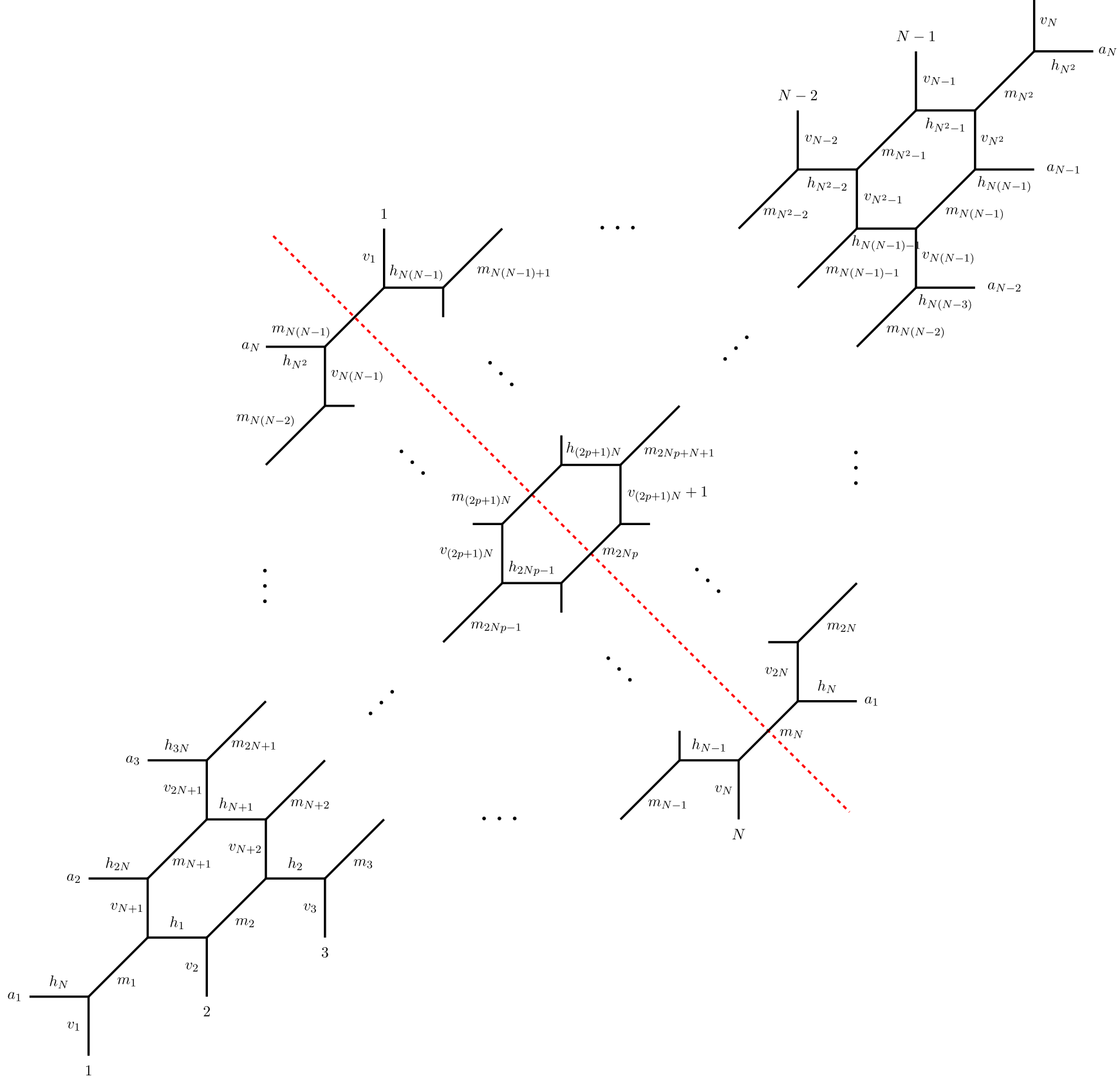


where the subscript $p=\left\lfloor\frac{N}{2}\right\rfloor$. Notice that not all of the $3 N^{2}$ parameters are independent due to the necessity of imposing linear constraints to guarantee the consistency of the web. Solving for these constraints, there are only $N^{2}+2$ independent parameters.

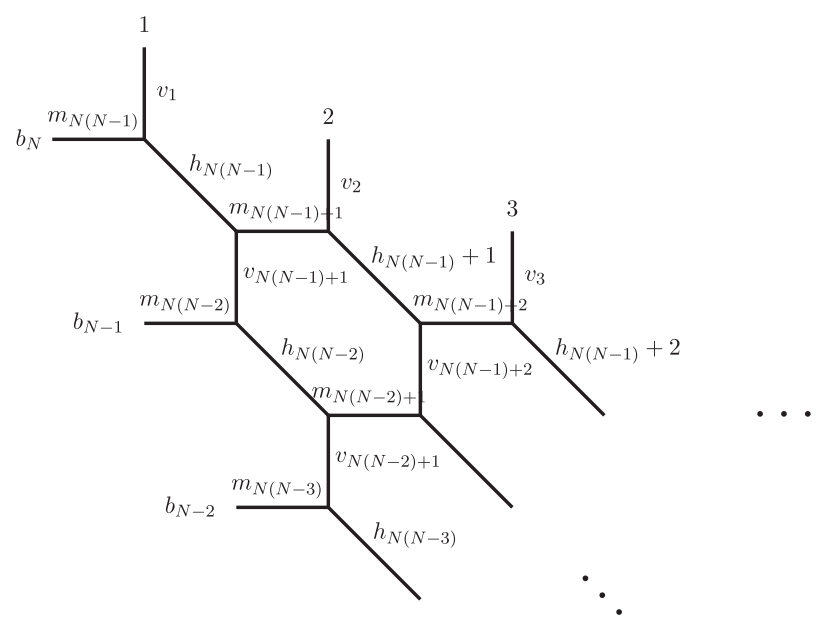

After cutting along the diagonal red line and re-gluing the diagram along the legs $a_{i}$ (for $i=1, \ldots, N$ ), we obtain the following equivalent web (after an appropriate $S L(2, \mathbb{Z})$-transformation that leaves the vertical lines invariant)

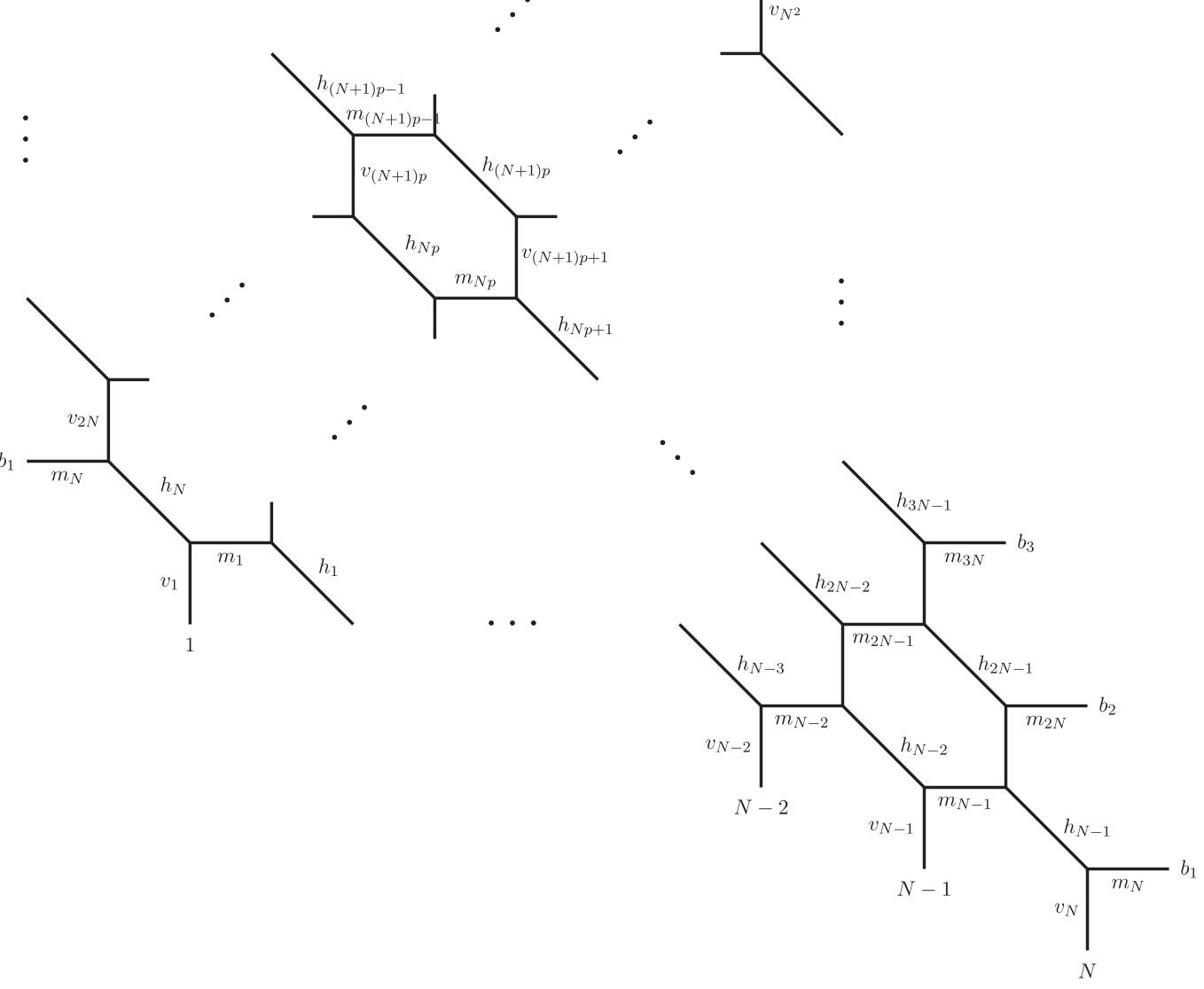

Here, the nodes $b_{i}$ indicate the gluing of the lines that have been cut in the previous step along the red line. As we can see, the diagram is again of the form of a $(N, N)$ web, except that the parameters $\{\mathbf{m}\}$ and $\{\mathbf{h}\}$ have been exchanged. This indicates, that the diagonal expansion also leads to a gauge theory with gauge group $U(N)^{N}$ where the mass parameters (from the perspective of the horizontal or vertical description) $\{\mathbf{m}\}$ are now related to the coupling constants. 
Finally, due to the known $S L(2, \mathbb{Z})$ duality $(N, M) \leftrightarrow$ $(M, N)$ of the compact brane configurations (i.e., those compactified on a torus), we can find a similar transformation that exchanges the parameters $\{\mathbf{m}\}$ and $\{\mathbf{v}\}$. As a result, we find a self-triality for the configurations $(N, N)$, given by the exchange of any of the three sets of parameters $\{\mathbf{h}\},\{\mathbf{v}\}$ or $\{\mathbf{m}\}$

$$
\{\mathbf{m}\} \leftrightarrow\{\mathbf{h}\} \leftrightarrow\{\mathbf{v}\} .
$$

Since the transformation described above transforms a $(N, N)$ web diagram into a $(N, N)$ web diagram, it is clear that (A2) is a symmetry of the partition function $\mathcal{Z}_{N, N}\left(\mathbf{h}, \mathbf{v}, \mathbf{m}, \epsilon_{1}, \epsilon_{2}\right)$ Eq. (2.20). As seen from the above figures, the precise map is somewhat complicated in the generic case. However, it can be made very explicit for specific examples: in the simplest case $(2,2)$, the transformation is shown in detail in the following figure

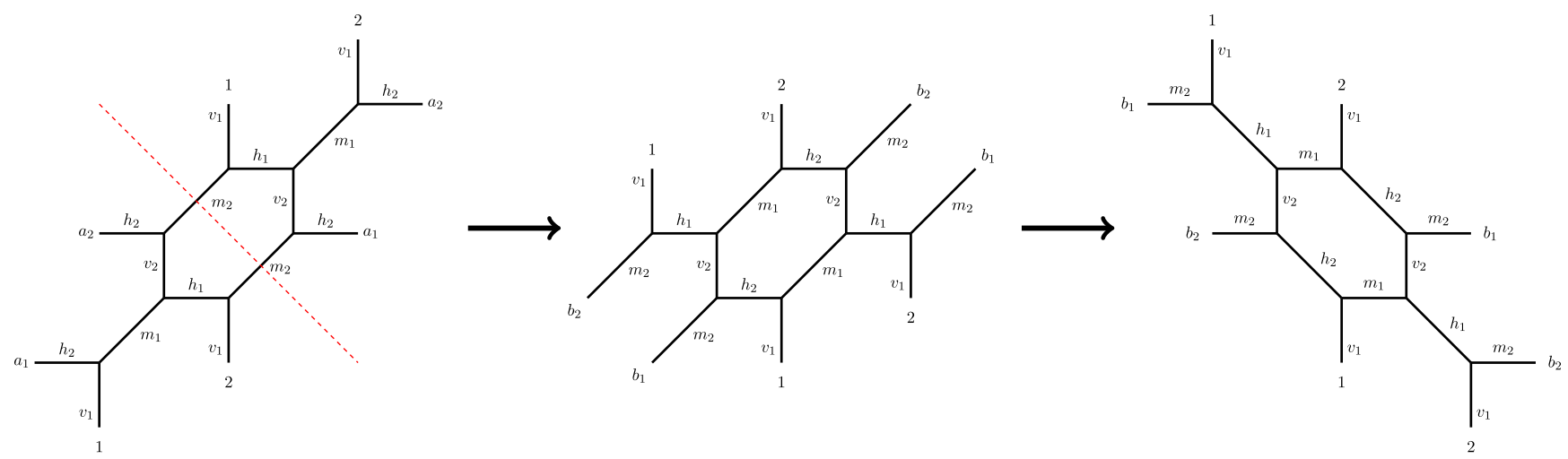

We see that the cutting and regluing procedure simply amounts to an exchange ${ }^{12}$ :

$$
\begin{aligned}
m_{1} & \rightarrow h_{1}, \\
h_{1} & \rightarrow m_{1}, \quad m_{2} \rightarrow h_{2}, \\
h_{2} & \rightarrow m_{2} .
\end{aligned}
$$

This indicates that the $(2,2)$ web indeed enjoys the triality symmetry

$$
\left(\begin{array}{l}
m_{1} \\
m_{2}
\end{array}\right) \leftrightarrow\left(\begin{array}{l}
h_{1} \\
h_{2}
\end{array}\right) \leftrightarrow\left(\begin{array}{l}
v_{1} \\
v_{2}
\end{array}\right) .
$$

\footnotetext{
${ }^{12}$ Notice that here the six parameters $\left(h_{1,2}, v_{1,2}, m_{1,2}\right)$ are all independent and satisfy the consistency conditions of the diagram.
}

\section{Configurations $(n M, M)$ with $n \in \mathbb{N}$}

The argument of the previous subsection for self-triality among the descriptions associated with the horizontal, vertical and diagonal expansions of $(N, N)$ configuration does not directly generalize to other generic $(M, N)$ configurations. However, for configurations $(N, M)$ with $N=n M$ for $n \in \mathbb{N}$ (and $n>1$ ), i.e., in the case that $N$ is an integer multiple of $M$, we find that the partition function is still invariant under the exchange of the parameters $\{\mathbf{v}\} \leftrightarrow\{\mathbf{m}\}$. While, in this case, there is no single cut like in the previous section that allows us to argue for this duality, the latter is still apparent from the Newton polygon of $X_{n M, M}$ given below. As is well known the Newton polygon is the dual graph of the web diagram of the toric Calabi-Yau threefold [49]. Indeed, for $N=n M$, the two fundamental domains in the latter, associated with the vertical and diagonal description, are as follows: 


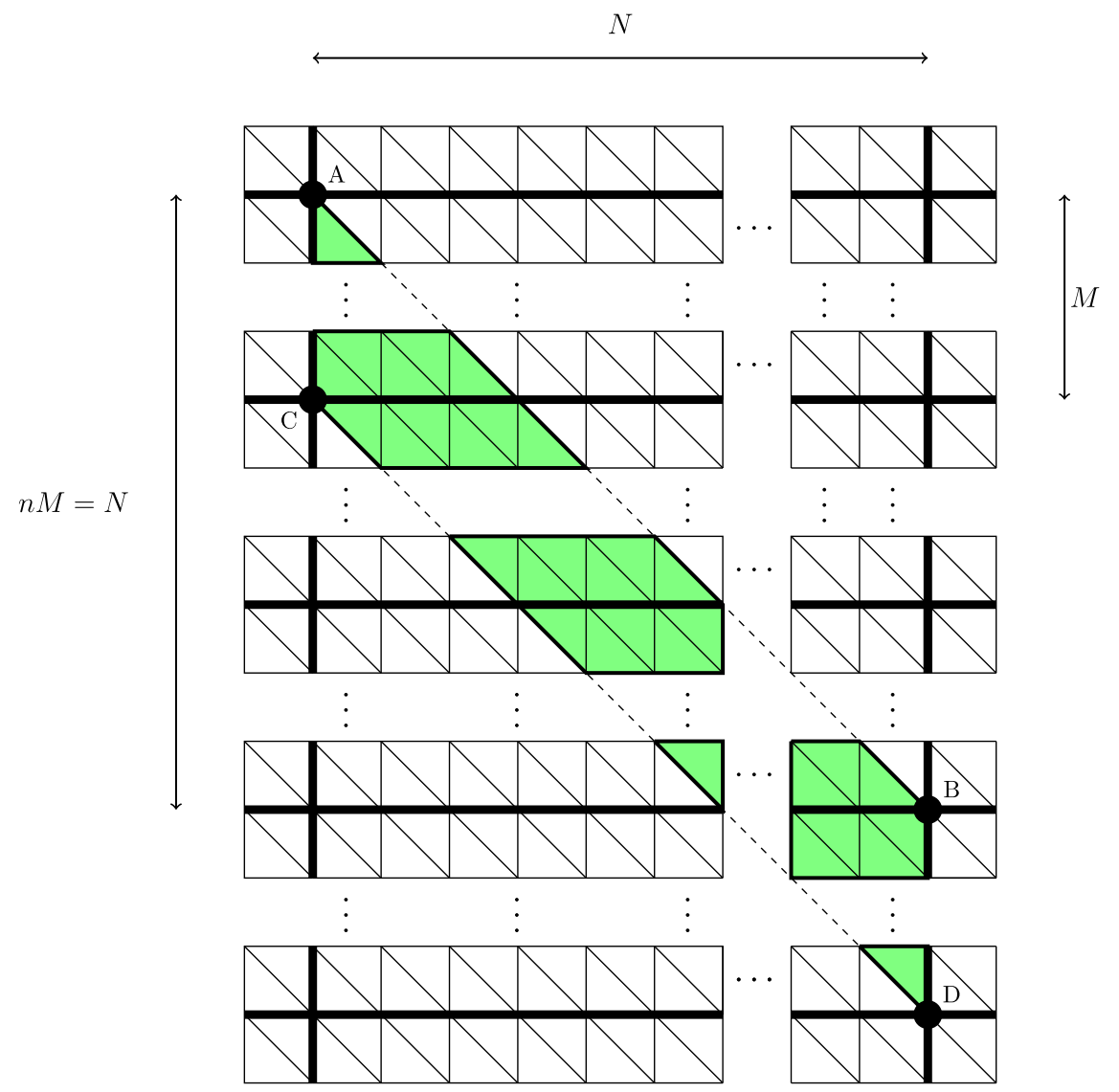

Here, the four points $A, B, C$ and $D$ are all equivalent, such that the diagonal lines $\overline{\mathrm{AB}}$ and $\overline{\mathrm{CD}}$ are identified with each other. Therefore, the web diagram dual to the green fundamental domain is again of the type $(n M, M)$, except that the diagonal parameters $\{\mathbf{m}\}$ are exchanged with the vertical ones $\{\mathbf{v}\}$. Thus, the theories $(n M, M)$ are self-dual under the exchange

$$
\{\mathbf{m}\} \leftrightarrow\{\mathbf{v}\} .
$$

For example, we can make this map more precise in the simplest case $M=2$ and $n=2$, i.e., for the configuration $(N, M)=(4,2)$, whose web diagram and Newton polygon are given by
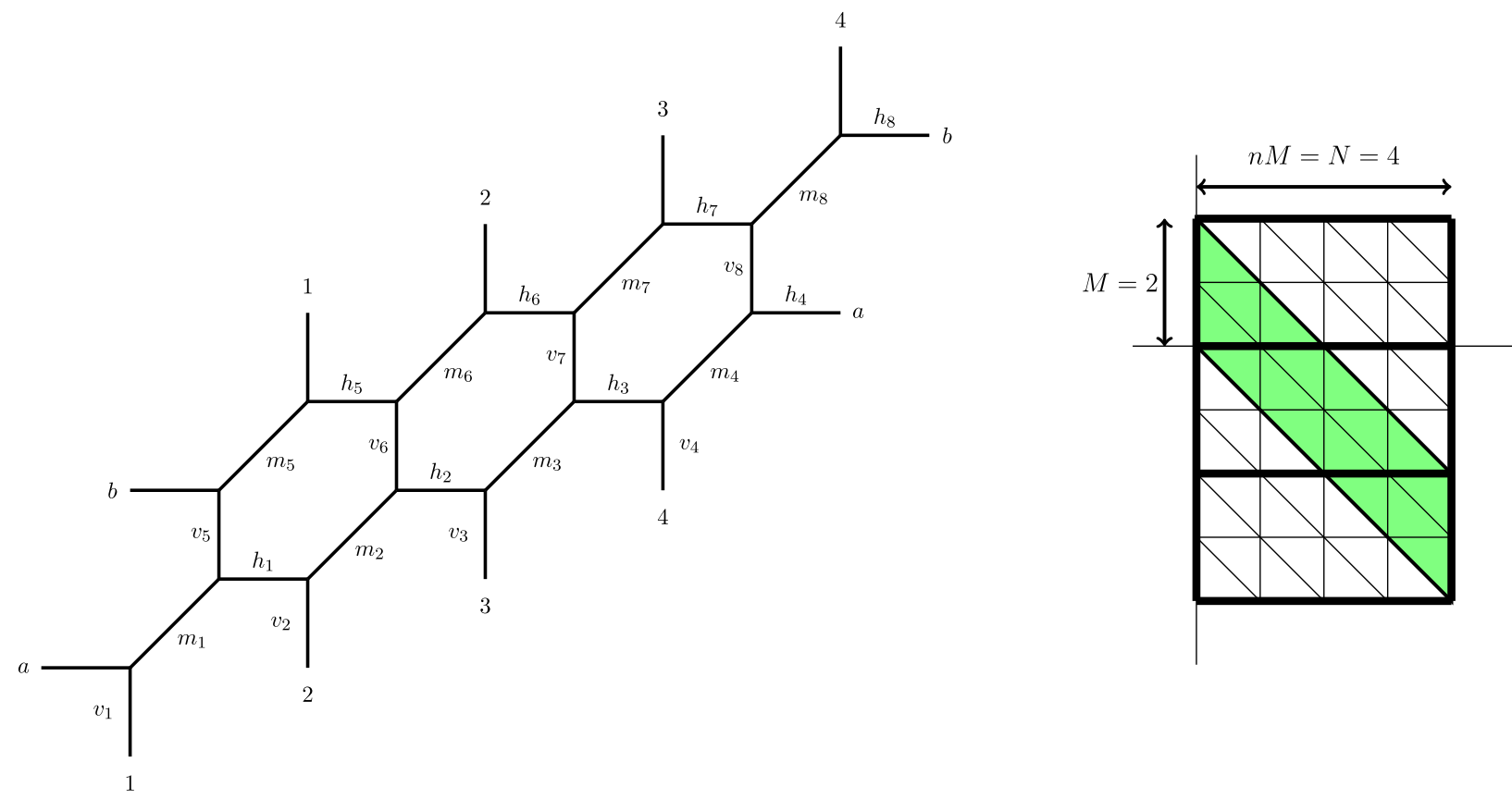


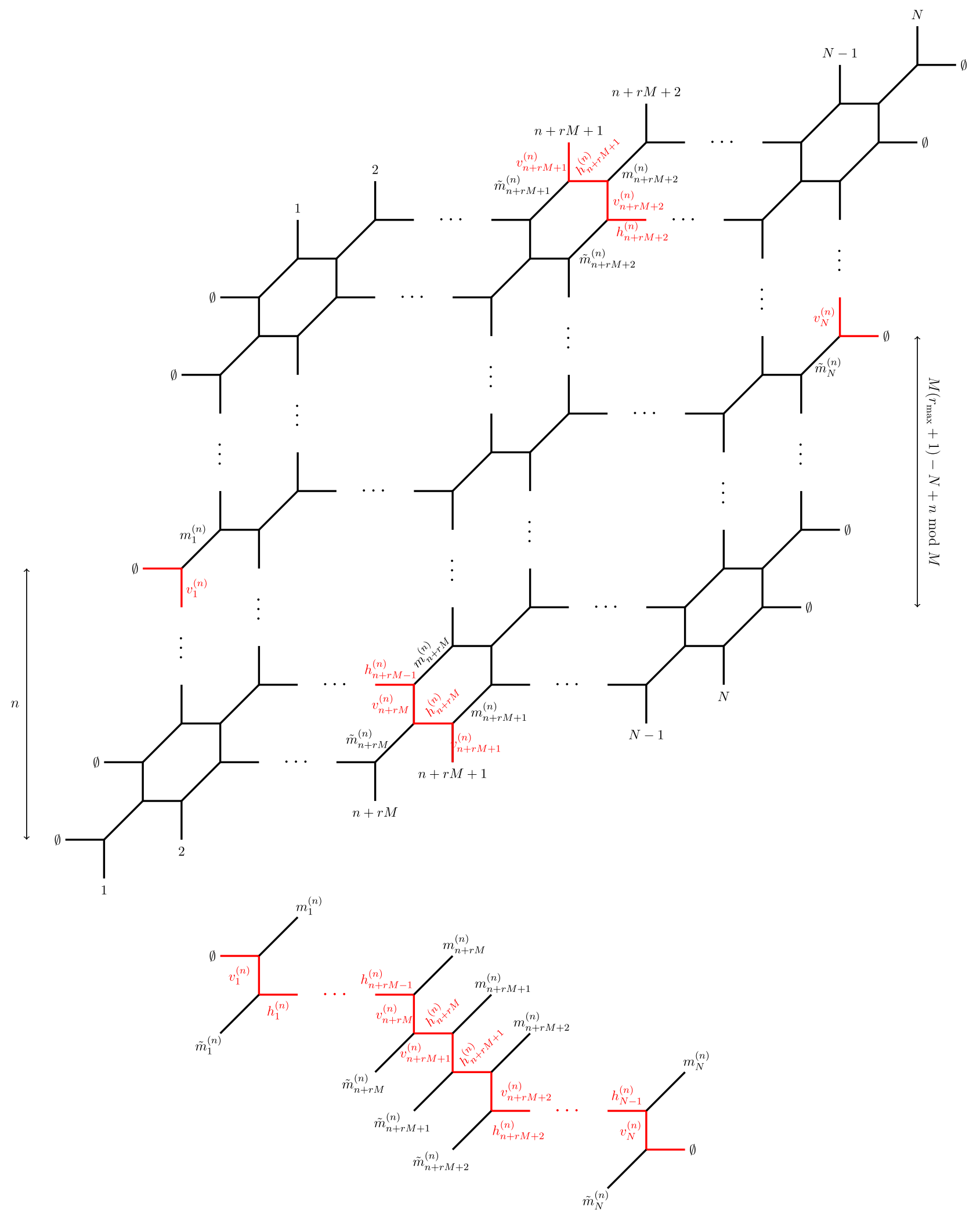

FIG. 9. Noncompact web diagram (top) with a generic strip (bottom) that runs between two lines stretching to infinity and is only composed of horizontal and vertical lines. 
This web diagram can also be presented in the form

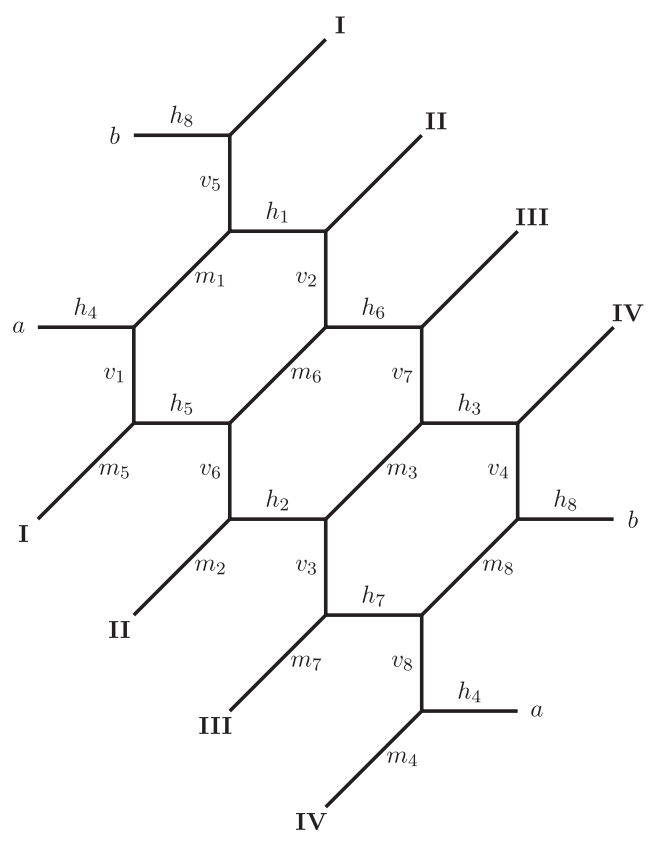

which is equivalent to the original diagram under the following exchange of parameters

$$
\begin{aligned}
& v_{1} \leftrightarrow m_{4}, \quad v_{2} \leftrightarrow m_{7}, \quad v_{3} \leftrightarrow m_{2}, \quad v_{4} \leftrightarrow m_{5}, \\
& v_{5} \leftrightarrow m_{8}, \quad v_{6} \leftrightarrow m_{3}, \quad v_{7} \leftrightarrow m_{6}, \quad v_{8} \leftrightarrow m_{1}, \\
& h_{1} \leftrightarrow h_{7}, \quad h_{3} \leftrightarrow h_{5},
\end{aligned}
$$

with $h_{2,4,6,8}$ remaining invariant. This makes the selfduality relation (A5) precise for the case $(4,2)$. Note, however, that these parameters are not independent one another, but instead the consistency conditions associated with the web diagram need to be imposed.

\section{Noncompact web diagrams}

Up to this point, we have studied $(N, M)$ web configurations that are defined on a torus (i.e., both the horizontal and vertical directions are periodic with radii $\rho$ and $\tau$, respectively). This setup defines the corresponding little string theories, as outlined in the introduction. It is also of interest to examine if various dualities we identified so far also hold for noncompact web configurations, where one of the two directions is decompactified by sending, e.g., $\rho \rightarrow i \infty$. These brane configurations, at a particular point in the moduli space, give rise to superconformal field theories. See [83-90] for a recent discussion of six-dimensional SCFTs.

It turns out that we can equally compute the equivalent of the diagonal expansion Eq. (2.21) while the $S L(2, \mathbb{Z})$ transformation maps between the vertical and diagonal expansion.

To show this, we consider a generic configuration of the type $(N, M)$ whose horizontal direction is decompactified and decompose it into $M$ strips of length $N$ that are glued together along the diagonal lines. A generic configuration of such strips (labeled by $n \in\{0, \ldots, M-1\}$ ) along with a labeling of the parameters involved is highlighted in Fig. 9, where the $\varnothing$ indicates that the horizontal lines stretch to infinity. Furthermore, for a given $n, r$ runs over all elements $\mathcal{S}_{n}=\{r \in \mathbb{N} \cup\{0\} \mid n+r M+1 \leq N\}$ and

$$
r_{\max }= \begin{cases}\max [\{r \in \mathbb{N} \cup\{0\} \mid n+r M+1 \leq N\}] & \text { if } \mathcal{S}_{n} \neq\{\} \\ -1 & \text { it } \mathcal{S}_{n}=\{\} .\end{cases}
$$
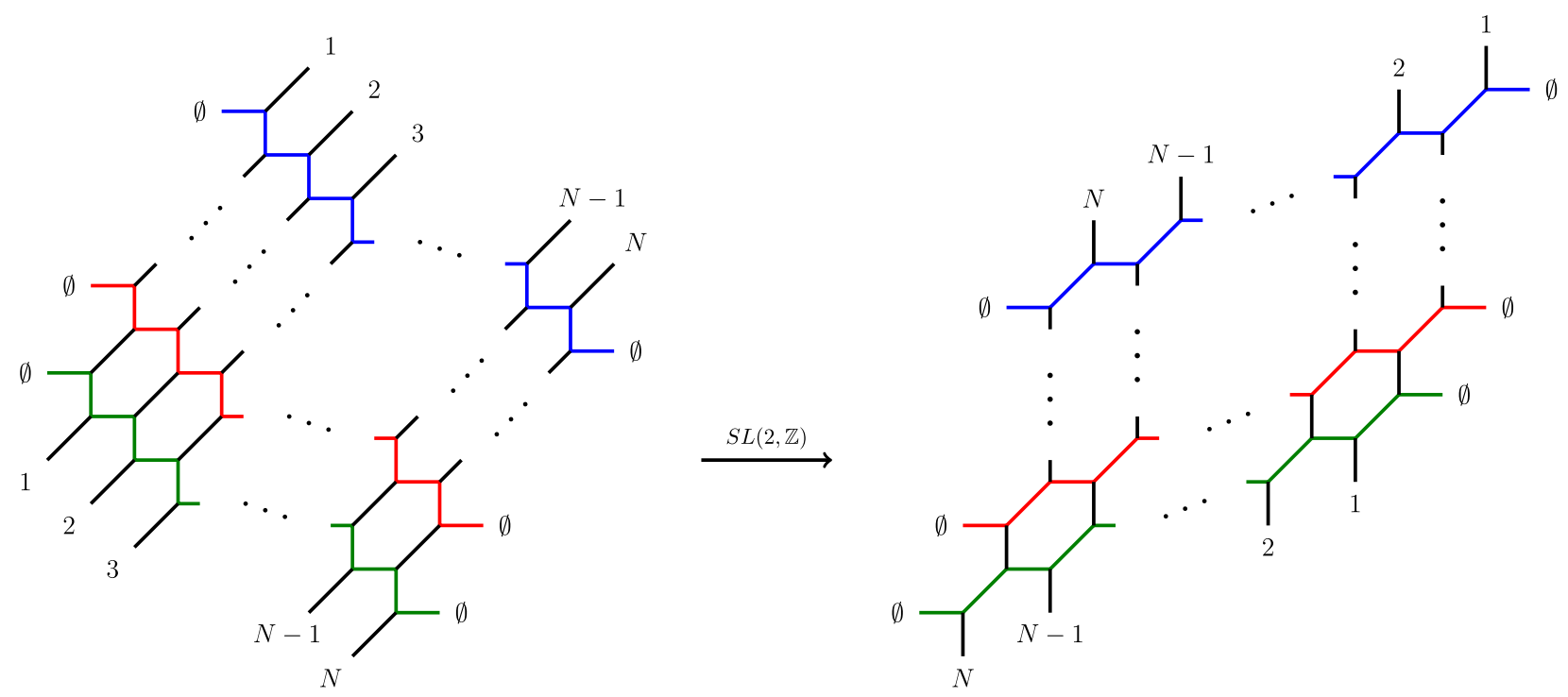

FIG. 10. Gluing the $M$ individual strips constructed in Fig. 9 yields another diagram of the type $(N, M)$. For better visibility, three different strips have been colored. 


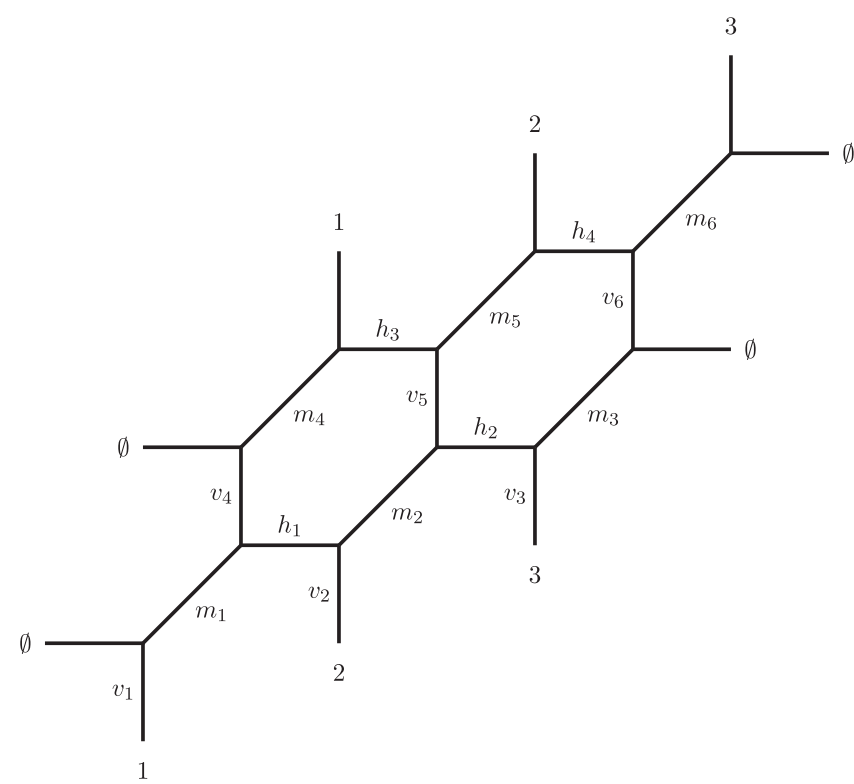

FIG. 11. Noncompact $(3,2)$ web diagram with a labeling of the Kähler parameters. Notice that the consistency conditions (A8)(A10) still need to be imposed.

Note that, for $\mathcal{S}_{n}=\{\}$, the strip never reaches the bottom of the diagram.

For two strips $\tilde{m}_{i}^{(n+1)}=m_{i}^{(n)}$ for $i=1, \ldots, N$ and $n=0, \ldots, M-1$, the $M$ strips are glued together to form another diagram that (with the help of an $S L(2, \mathbb{Z})$ transformation) can be brought into the form of a $(N, M)$ web, as shown in Fig. 10. However, comparing the two diagrams, we see that the role of diagonal and vertical lines is exchanged, leading to the self-duality

$$
\{\mathbf{m}\} \leftrightarrow\{\mathbf{v}\} .
$$

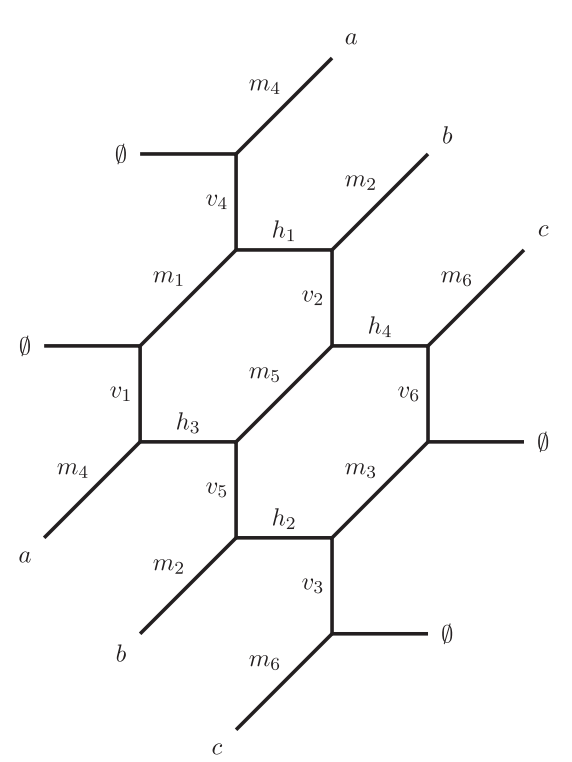

The precise duality map requires also to impose the consistency conditions, which can be worked out explicitly for specific examples. As an example, we may consider the noncompact $(N, M)=(3,2)$ configuration, whose web diagram (along with a labeling of the various lines) is given by Fig. 11. In this configuration, the consistency conditions for the various Kähler parameters are

$$
\begin{aligned}
& m_{1}+h_{1}=h_{3}+m_{5}, v_{1}+m_{1}=m_{5}+v_{2}, \\
& m_{2}+h_{2}=h_{4}+m_{6}, v_{2}+m_{2}=v_{3}+m_{6}, \\
& h_{1}+m_{2}=m_{4}+h_{3}, v_{4}+m_{4}=m_{2}+v_{5}, \\
& h_{2}+m_{3}=m_{5}+h_{4}, v_{5}+m_{5}=m_{3}+v_{6}, \\
& v_{1}+m_{1}+v_{4}+m_{4}=v_{2}+m_{2}+v_{5}+m_{5} \\
&=v_{3}+m_{3}+m_{6}+v_{6} .
\end{aligned}
$$

This web diagram can represented in an equivalent fashion as shown in Fig. 12. Under an $S L(2, \mathbb{Z})$ transformation, this web can again be presented in the form of a $(3,2)$ configuration which is dual to the original configuration upon the change of variables

$$
\begin{aligned}
& m_{1} \rightarrow v_{3}, \quad m_{1} \rightarrow v_{3}, \quad m_{3} \rightarrow v_{1}, \\
& m_{4} \rightarrow v_{6}, \quad m_{5} \rightarrow v_{2}, \quad m_{6} \rightarrow v_{4}, \\
& v_{1} \rightarrow m_{6}, \quad v_{2} \rightarrow m_{2}, \quad v_{3} \rightarrow m_{4}, \\
& v_{4} \rightarrow m_{3}, \quad v_{5} \rightarrow m_{5}, \quad v_{6} \rightarrow m_{1}, \\
& h_{1} \rightarrow h_{2}, \quad h_{2} \rightarrow h_{3}, \quad h_{3} \rightarrow h_{4}, \quad h_{4} \rightarrow h_{1} \text {. }
\end{aligned}
$$

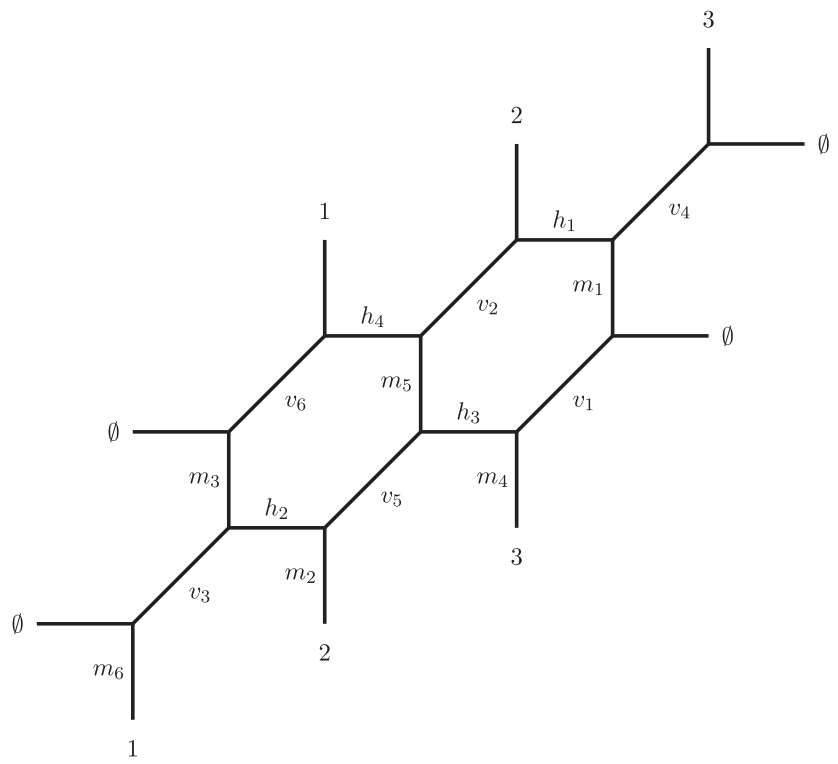

FIG. 12. Different representation of the $(3,2)$ diagram (left) and dual diagram after an $S L(2, \mathbb{Z})$ transformation (right). 
[1] N. Seiberg and E. Witten, Comments on string dynamics in six dimensions, Nucl. Phys. B471, 121 (1996).

[2] M. Berkooz, M. Rozali, and N. Seiberg, Matrix description of M theory on $T^{4}$ and $T^{5}$, Phys. Lett. B 408, 105 (1997).

[3] J. D. Blum and K. A. Intriligator, New phases of string theory and 6-D RG fixed points via branes at orbifold singularities, Nucl. Phys. B506, 199 (1997).

[4] N. Seiberg, New theories in six dimensions and matrix description of $\mathrm{M}$ theory on $T^{5}$ and $T^{5} / Z_{2}$, Phys. Lett. B 408, 98 (1997).

[5] A. Losev, G. W. Moore, and S. L. Shatashvili, M \& m's, Nucl. Phys. B522, 105 (1998).

[6] K. A. Intriligator, New string theories in six dimensions via branes at orbifold singularities, Adv. Theor. Math. Phys. 1, 271 (1997).

[7] O. Aharony, M. Berkooz, D. Kutasov, and N. Seiberg, Linear dilatons, NS five-branes and holography, J. High Energy Phys. 10 (1998) 004.

[8] O. Aharony, A brief review of "little string theories," Classical Quantum Gravity 17, 929 (2000).

[9] D. Kutasov, Introduction to little string theory, in Spring School on Superstrings and Related Matters (ICTP, 2001), pp. 165-209.

[10] L. Bhardwaj, M. D. Zotto, J. J. Heckman, D. R. Morrison, T. Rudelius, and C. Vafa, F-theory and the classification of little strings, Phys. Rev. D 93, 086002 (2016).

[11] S. Hohenegger, A. Iqbal, and S. J. Rey, Dual little strings from F-theory and flop transitions, J. High Energy Phys. 07 (2017) 112.

[12] S. Hohenegger, A. Iqbal, and S. J. Rey, Self-duality and selfsimilarity of little string orbifolds, Phys. Rev. D 94, 046006 (2016).

[13] B. Haghighat, G. Lockhart, and C. Vafa, Fusing E-strings to heterotic strings: $E+E \mapsto H$, Phys. Rev. D 90, 126012 (2014).

[14] K. S. Choi and S. J. Rey, E(lementary) strings in sixdimensional heterotic F-theory, J. High Energy Phys. 09 (2017) 092.

[15] K. S. Choi and S. J. Rey, Elliptic genus, anomaly cancellation and heterotic M-theory, arXiv:1710.07627.

[16] D. R. Morrison and C. Vafa, Compactifications of F theory on Calabi-Yau threefolds, Nucl. Phys. B473, 74 (1996).

[17] D. R. Morrison and C. Vafa, Compactifications of F theory on Calabi-Yau threefolds. 2., Nucl. Phys. B476, 437 (1996).

[18] M. Bershadsky and A. Johansen, Colliding singularities in F theory and phase transitions, Nucl. Phys. B489, 122 (1997).

[19] P. S. Aspinwall, Point-like instantons and the $\operatorname{spin}(32) / \mathbb{Z}_{2}$ heterotic string, Nucl. Phys. B496, 149 (1997).

[20] M. Bershadsky and C. Vafa, Global anomalies and geometric engineering of critical theories in six dimensions, arXiv:hep-th/9703167.

[21] M. Esole and S. H. Shao, M-theory on elliptic Calabi-Yau threefolds and 6d anomalies, arXiv:1504.01387.

[22] S. Hohenegger, A. Iqbal, and S. J. Rey, Instanton-monopole correspondence from M-branes on $\mathbb{S}^{1}$ and little string theory, Phys. Rev. D 93, 066016 (2016).

[23] A. Kanazawa and S. C. Lau, Local Calabi-Yau manifolds of affine type A and open Yau-Zaslow formula via SYZ mirror symmetry, arXiv:1605.00342.
[24] J. D. Blum and K. A. Intriligator, Consistency conditions for branes at orbifold singularities, Nucl. Phys. B506, 223 (1997).

[25] S. Cecotti, D. Gaiotto, and C. Vafa, $t t^{*}$ geometry in 3 and 4 dimensions, J. High Energy Phys. 05 (2014) 055.

[26] B. Haghighat, A. Iqbal, C. Kozçaz, G. Lockhart, and C. Vafa, M-strings, Commun. Math. Phys. 334, 779 (2015).

[27] B. Haghighat, C. Kozcaz, G. Lockhart, and C. Vafa, Orbifolds of M-strings, Phys. Rev. D 89, 046003 (2014).

[28] A. Iqbal, C. Kozcaz, and C. Vafa, The refined topological vertex, J. High Energy Phys. 10 (2009) 069.

[29] S. Hohenegger and A. Iqbal, M-strings, elliptic genera and $\mathcal{N}=4$ string amplitudes, Fortsch. Phys. 62, 155 (2014).

[30] N. A. Nekrasov, Seiberg-Witten prepotential from instanton counting, Adv. Theor. Math. Phys. 7, 831 (2003).

[31] A. S. Losev, A. Marshakov, and N. A. Nekrasov, Small instantons, little strings and free fermions, in From Fields to Strings: Circumnavigating Theoretical Physics, edited by M. Shifman, A. Vainshtein, and J. F. Wheater (World Scientific, 2004), Vol. 1, pp. 581-621.

[32] T. J. Hollowood, A. Iqbal, and C. Vafa, Matrix models, geometric engineering and elliptic genera, J. High Energy Phys. 03 (2008) 069.

[33] B. Bastian and S. Hohenegger, Five-brane webs and highest weight representations, J. High Energy Phys. 12 (2017) 020.

[34] A. Klemm, J. Manschot, and T. Wotschke, Quantum geometry of elliptic Calabi-Yau manifolds, arXiv: 1205.1795.

[35] M. X. Huang, S. Katz, and A. Klemm, Topological string on elliptic CY 3-folds and the ring of Jacobi forms, J. High Energy Phys. 10 (2015) 125.

[36] B. Bastian, S. Hohenegger, A. Iqbal, and S. J. Rey, Dual little strings and their partition functions, arXiv:1710.02455.

[37] L. F. Alday, D. Gaiotto, and Y. Tachikawa, Liouville correlation functions from four-dimensional gauge theories, Lett. Math. Phys. 91, 167 (2010).

[38] N. Wyllard, $A_{N-1}$ conformal Toda field theory correlation functions from conformal $N=2, S U(N)$ quiver gauge theories, J. High Energy Phys. 11 (2009) 002.

[39] H. Awata and Y. Yamada, Five-dimensional AGT conjecture and the deformed Virasoro algebra, J. High Energy Phys. 01 (2010) 125.

[40] R. Schiappa and N. Wyllard, An $A(r)$ threesome: Matrix models, 2d CFTs and $4 d, N=2$ gauge theories, J. Math. Phys. 51, 082304 (2010).

[41] H. Awata and Y. Yamada, Five-dimensional AGT relation and the deformed beta-ensemble, Prog. Theor. Phys. 124, 227 (2010).

[42] T. Kimura and V. Pestun, Quiver W-algebras, arXiv: 1512.08533.

[43] B. Bastian, S. Hohenegger, A. Iqbal, and S.-J. Rey (Work in progress).

[44] E. Witten, Some comments on string dynamics, in Future Perspectives in String Theory Strings 1995, edited by I. Bars, P. Bouwknegt, J. Minahan, D. Nemeschansky, K. Pilch, H. Saleur, and N. Warner (World Scientific, 1997), p. 501.

[45] E. Witten, Small instantons in string theory, Nucl. Phys. B460, 541 (1996).

[46] A. Strominger, Open p-branes, Phys. Lett. B 383, 44 (1996). 
[47] O.J. Ganor and A. Hanany, Small E(8) instantons and tensionless noncritical strings, Nucl. Phys. B474, 122 (1996).

[48] N. Seiberg, Nontrivial fixed points of the renormalization group in six-dimensions, Phys. Lett. B 390, 169 (1997).

[49] N. C. Leung and C. Vafa, Branes and toric geometry, Adv. Theor. Math. Phys. 2, 91 (1998).

[50] A. Ahmed, S. Hohenegger, A. Iqbal, and S. J. Rey, Bound states of little strings and symmetric orbifold CFTs, Phys. Rev. D 96, 081901 (2017).

[51] G. W. Moore, N. Nekrasov, and S. Shatashvili, Integrating over Higgs Branches, Commun. Math. Phys. 209, 97 (2000).

[52] A. Losev, N. Nekrasov, and S. L. Shatashvili, Testing Seiberg-Witten solution, in Proceedings of the NATO Advanced Study Institute on Strings, Branes and Dualities Cargese, France, 1997 (Springer, 1997), p. 359.

[53] E. Witten, Phase transitions in M theory and F theory, Nucl. Phys. B471, 195 (1996).

[54] S. Ferrara, R. Minasian, and A. Sagnotti, Low-energy analysis of $\mathrm{M}$ and $\mathrm{F}$ Theories on Calabi-Yau threefolds, Nucl. Phys. B474, 323 (1996).

[55] A. S. Chou, R. Kallosh, J. Rahmfeld, S. J. Rey, M. Shmakova, and W. K. Wong, Critical points and phase transitions in 5-D compactifications of $\mathrm{M}$ theory, Nucl. Phys. B508, 147 (1997).

[56] K. A. Intriligator, D. R. Morrison, and N. Seiberg, Fivedimensional supersymmetric gauge theories and degenerations of Calabi-Yau spaces, Nucl. Phys. B497, 56 (1997).

[57] A. Iqbal and A. K. Kashani-Poor, Instanton counting and Chern-Simons theory, Adv. Theor. Math. Phys. 7, 457 (2003).

[58] A. Iqbal and A. K. Kashani-Poor, SU(N) geometries and topological string amplitudes, Adv. Theor. Math. Phys. 10, 1 (2006).

[59] M. Bershadsky, S. Cecotti, H. Ooguri, and C. Vafa, KodairaSpencer theory of gravity and exact results for quantum string amplitudes, Commun. Math. Phys. 165, 311 (1994).

[60] Y. Tachikawa, Five-dimensional Chern-Simons terms and Nekrasov's instanton counting, J. High Energy Phys. 02 (2004) 050.

[61] R. Gopakumar and C. Vafa, M theory and topological strings. 1., arXiv:hep-th/9809187.

[62] R. Gopakumar and C. Vafa, M Theory and topological strings. 2., arXiv:hep-th/9812127.

[63] M. Taki, Refined Topological Vertex and Instanton Counting, J. High Energy Phys. 03 (2008) 048.

[64] E. Witten, Solutions of four-dimensional field theories via M theory, Nucl. Phys. B500, 3 (1997).

[65] D. Gaiotto, $N=2$ dualities, J. High Energy Phys. 08 (2012) 034.

[66] R. Dijkgraaf and C. Vafa, Toda theories, matrix models, topological strings, and $N=2$ gauge systems, arXiv: 0909.2453 .

[67] H. Nakajima and K. Yoshioka, Instanton counting on blowup. II. K-theoretic partition function, arXiv:math/0505553.

[68] T. Kimura, Double quantization of Seiberg-Witten geometry and W-algebras, in Proceedings of the 2016 von Neumann Symposium on Topological Recursion and its Influence in Analysis, Geometry, and Topology, 4-8 July 2016, Charlotte, NC, arXiv:1612.07590.

[69] M.-C. Tan, An M-theoretic derivation of a 5d and 6d AGT correspondence, and relativistic and elliptized integrable systems, J. High Energy Phys. 12 (2013) 031.

[70] A. Iqbal, C. Kozcaz, and S. T. Yau, Elliptic Virasoro conformal blocks, arXiv:1511.00458.

[71] F. Nieri, An elliptic Virasoro symmetry in 6d, Lett. Math. Phys. 107, 2147 (2017).

[72] M.-C. Tan, Higher AGT correspondences, W-algebras, and higher quantum geometric Langlands duality from M-theory, arXiv:1607.08330.

[73] T. Kimura and V. Pestun, Quiver elliptic W-algebras, arXiv:1608.04651.

[74] D. Maulik and A. Okounkov, Quantum groups and quantum cohomology, arXiv:1211.1287.

[75] O. Schiffmann and E. Vasserot, Cherednik algebras, $\mathrm{W}$-algebras and the equivariant cohomology of the moduli space of instantons on $A_{2}$, Publ. Math. Inst. Hautes Etudes Sci. 118, 213 (2013).

[76] A. Braverman, M. Finkelberg, and H. Nakajima, Instanton moduli spaces and $W$-algebras, arXiv:1406.2381.

[77] M. Aganagic, E. Frenkel, and A. Okounkov, Quantum q-Langlands correspondence, arXiv:1701.03146.

[78] N. Nekrasov and V. Pestun, Seiberg-Witten geometry of four dimensional $N=2$ quiver gauge theories, arXiv: 1211.2240.

[79] S. Katz, P. Mayr, and C. Vafa, Mirror symmetry and exact solution of $4-D N=2$ gauge theories: 1., Adv. Theor. Math. Phys. 1, 53 (1997).

[80] L. Bao, E. Pomoni, M. Taki, and F. Yagi, M5-branes, toric diagrams and gauge theory duality, J. High Energy Phys. 04 (2012) 105.

[81] M. Aganagic, N. Haouzi, C. Kozcaz, and S. Shakirov, Gauge/Liouville triality, arXiv:1309.1687.

[82] M. Aganagic and N. Haouzi, ADE little string theory on a Riemann surface (and triality), arXiv:1506.04183.

[83] J. J. Heckman, D. R. Morrison, and C. Vafa, On the classification of 6D SCFTs and generalized ADE orbifolds, J. High Energy Phys. 05 (2014) 028; Erratum, J. High Energy Phys. 06 (2015) 17(E).

[84] J. J. Heckman, More on the matter of 6D SCFTs, Phys. Lett. B 747, 73 (2015).

[85] K. Ohmori, H. Shimizu, Y. Tachikawa, and K. Yonekura, Anomaly polynomial of general 6d SCFTs, Prog. Theor. Exp. Phys. 10 (2014) 103B07.

[86] B. Haghighat, A. Klemm, G. Lockhart, and C. Vafa, Strings of minimal 6d SCFTs, Fortsch. Phys. 63, 294 (2015).

[87] M. D. Zotto, J. J. Heckman, A. Tomasiello, and C. Vafa, 6d conformal matter, J. High Energy Phys. 02 (2015) 054.

[88] J. J. Heckman, D. R. Morrison, T. Rudelius, and C. Vafa, Atomic classification of 6D SCFTs, Fortsch. Phys. 63, 468 (2015).

[89] M. Bertolini, P. R. Merkx, and D. R. Morrison, On the global symmetries of 6D superconformal field theories, J. High Energy Phys. 07 (2016) 005.

[90] M. D. Zotto, J. J. Heckman, and D. R. Morrison, 6D SCFTs and phases of 5D theories, J. High Energy Phys. 09 (2017) 147. 